\title{
QUASI-PARABOLIC INITIAL-BOUNDARY VALUE PROBLEMS
}

\author{
VEIKKO T. PURMONEN
}

\section{INTRODUCTION}

Let $A(\partial / \partial t, D)$ and $B_{j}(\partial / \partial t, D), j=1, \ldots, x$, be linear partial differential operators with complex constant coefficients or, as we shall simply say, differential operators in $\boldsymbol{R}_{t} \times \boldsymbol{R}^{n}$. This paper is concerned with the following initial-boundary problem:

$$
\begin{array}{lll}
A(\partial / \partial t, D) u=f & \text { in } & \boldsymbol{R}_{+} \times \boldsymbol{R}_{+}^{n}, \\
\left.\frac{\partial^{k} u}{\partial t^{k}}\right|_{t=0}=\varphi_{k} & \text { on } & \boldsymbol{R}_{+}^{n}, \\
\left.B_{j}(\partial / \partial t, D) u\right|_{x_{n}=0}=g_{j} & \text { on } & \boldsymbol{R}_{+} \times \boldsymbol{R}^{n-1} .
\end{array}
$$

We shall investigate the existence and uniqueness of solutions to the problem (1)-(3), posed in suitable function spaces, as well as the validity of a priori estimates of the form (see Section 12)

$$
\|u\| \leqq C\left(\|f\|+\left\|\left(\left(\Phi_{k}\right)_{k},\left(g_{j}\right)_{j}\right)\right\|\right)
$$

with the corresponding norms; note that throughout the paper $C$ is used to denote a generic positive constant.

The idea of our treatment is in brief as follows: First, we make use of the Laplace transformation with respect to $t$ to transform the problem (1)-(3) with $\varphi_{k}=0$ and $g_{j}=0$ into a parametrically quasi-elliptic boundary problem

$$
\begin{array}{ll}
A(z, D) U=F & \text { in } \quad \boldsymbol{R}_{+}^{n}, \\
\left.B_{j}(z, D) U\right|_{x_{n}=0}=0 & \text { on } \boldsymbol{R}^{n-1},
\end{array}
$$

where $z \in \boldsymbol{C}$ with $\operatorname{Re} z \geqq 0$. Second, in order to handle boundary problems of this type we exploit the results of our previous paper [6]. Third, by means of detailed analysis of the initial and boundary values of functions defined on $\boldsymbol{R}_{+} \times \boldsymbol{R}_{+}^{n}$, we are able to treat the problem (1)-(3) in the general case. 
M. S. Agranovič and M. I. Višik have investigated in [1] the classical parabolic problem for which the boundary problem

$$
\begin{array}{ll}
A(z, D) U=F & \text { in } \boldsymbol{R}_{+}^{n}, \\
\left.B_{j}(z, D) U\right|_{x_{n}=0}=G_{j} & \text { on } \boldsymbol{R}^{n-1},
\end{array}
$$

is elliptic for every $z \in \boldsymbol{C}$ with $\operatorname{Re} z \geqq 0$. We shall therefore find it natural and motivated to call our problem quasi-parabolic.

Besides this introductory chapter, which also includes the first two numbered sections, there are three chapters.

The first deals with anisotropic Sobolev spaces, in Sections 3 and 4, and with Hilbert spaces of holomorphic functions, in Sections 5 and 6. It will turn out that these spaces form, from our point of view, a natural framework for the study of the problem (1)-(3).

In the next chapter we give first a result concerning initial values in Section 7. In Section 8 we then discuss the compatibility of initial and boundary values; the results are related to those of $\mathrm{P}$. Grisvard [2].

The last chapter is devoted to the realization of the plan sketched above. In Section 9 we obtain an a priori estimate in the case of homogeneous initial values. The existence and uniqueness of solutions for homogeneous initial and boundary values will be proved in Section 10. In each of these sections we make essential use of the results of [6]. In the general case we first give a necessary and sufficient condition for the existence of solutions in Section 11. The uniqueness of solutions is also proved. In Section 12 we then study the validity of a priori estimates and obtain a condition which turns out to be both necessary and sufficient. Finally, it is illustrative to consider the special case of normal boundary operator systems $\left\{B_{j}(D)\right\}$. This is done in Section 13 , and we shall see that the necessary and sufficient condition for the problem (1)-(3) to have a solution takes now a very concrete form. In addition, it will be shown that the condition obtained in Section 12 holds; hence an a priori estimate of the form (4) is also valid. We hope to discuss the general case of normal boundary operator systems $\left\{B_{j}(\partial / \partial t, D)\right\}$ at a future time.

Acknowledgement. For financial support I am indebted to the Emil Aaltonen Foundation.

\section{Contents}

Introduction

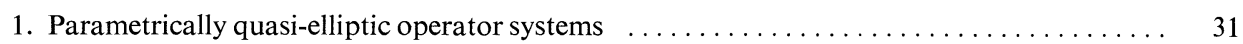

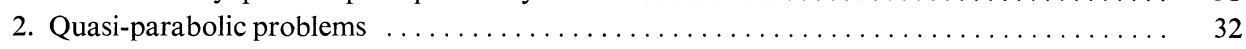

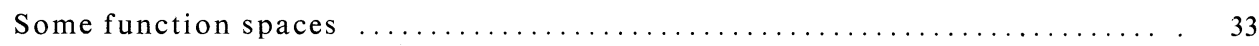

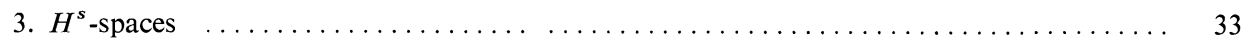

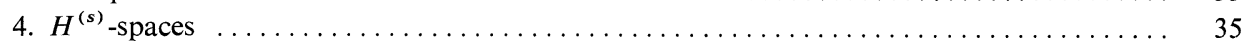




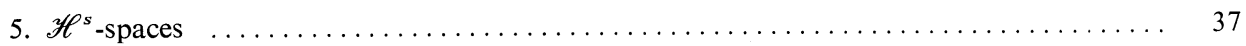

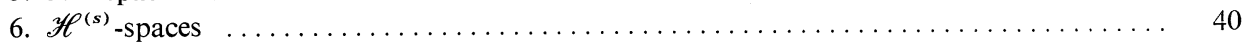

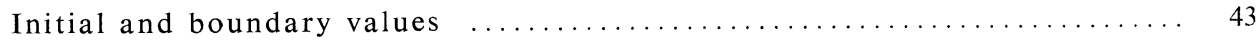

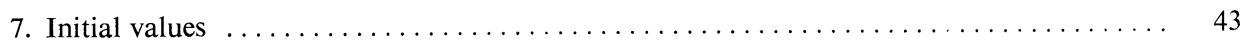

8. Initial and boundary values. The spaces $G^{s}(\varrho), F^{s}(\varrho)$, and $E^{s}(\varrho) \ldots \ldots \ldots \ldots \ldots$

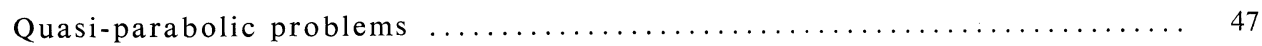

9. A priori estimates in $\mathscr{H}^{(s)}\left(\boldsymbol{C}_{\varrho}, \boldsymbol{R}_{+}^{n}\right)$ and $H_{(0)}^{(s)}(Q ; \varrho) \ldots \ldots \ldots \ldots \ldots \ldots \ldots \ldots$

10. Existence and uniqueness of solutions for homogeneous initial and boundary values . . . 49

11. Existence and uniqueness theorem in the general case $\ldots \ldots \ldots \ldots \ldots \ldots \ldots \ldots \ldots \ldots \ldots \ldots$

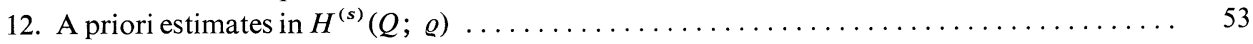

13. The case of normal boundary operator system $\left\{B_{j}(D)\right\} \ldots \ldots \ldots \ldots \ldots \ldots \ldots \ldots$

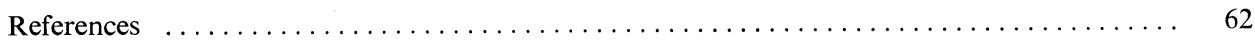

\section{Parametrically quasi-elliptic operator systems}

1.1. Let $\tau \in \boldsymbol{R}=\boldsymbol{R}_{\tau}$ denote the dual variable of $t \in \boldsymbol{R}=\boldsymbol{R}_{t}$ and $\xi=\left(\xi^{\prime}, \xi_{n}\right)=$ $\left(\xi_{1}, \ldots, \xi_{n-1}, \xi_{n}\right) \in \boldsymbol{R}^{n}=\boldsymbol{R}_{\xi}^{n}$ the one of $x=\left(x^{\prime}, x_{n}\right)=\left(x_{1}, \ldots, x_{n-1}, x_{n}\right) \in \boldsymbol{R}^{n}=\boldsymbol{R}_{x}^{n}$, and set

$$
\langle x, \xi\rangle=x_{1} \xi_{1}+\ldots+x_{n} \xi_{n}
$$

Let $m_{k}, k=0,1, \ldots, n$, be positive integers, $\mu=\max \left\{m_{k}\right\}, q_{k}=\mu / m_{k}, \quad$ and $q=\left(q_{1}, \ldots, q_{n}\right)$.

We set

and

$$
\begin{aligned}
\left\langle\xi^{\prime}\right\rangle & =\left(\sum_{k=1}^{n-1}\left|\xi_{k}\right| m_{k}\right)^{1 / \mu}, \\
\left\langle\xi_{n}\right\rangle & =\left|\xi_{n}\right|^{1 / q_{n}}, \\
\langle\xi\rangle & =\left(\left\langle\xi^{\prime}\right\rangle^{\mu}+\left\langle\xi_{n}\right\rangle^{\mu}\right)^{1 / \mu},
\end{aligned}
$$

$$
\langle w\rangle=|w|^{1 / q_{0}},
$$

where $w$ stands for $\tau \in \boldsymbol{R}$ or for a complex number $z=\operatorname{Re} z+i \operatorname{Im} z \in \boldsymbol{C}$; set also for $z \in \boldsymbol{C}$ and for $\eta=\xi^{\prime}$ or $\xi$.

$$
h(z, \eta)=\langle z\rangle+\langle\eta\rangle
$$

Let $D_{k}$ be the operator $-i \partial / \partial x_{k}, k=1, \ldots, n, D=\left(D_{1}, \ldots, D_{n}\right)$, and set

$$
D^{\alpha}=D_{1}^{\alpha_{1}} \ldots D_{n}^{\alpha_{n}}, \quad \xi^{\alpha}=\xi_{1}^{\alpha_{1}} \ldots \xi_{n}^{\alpha_{n}}
$$

for every multi-index $\alpha=\left(\alpha_{1}, \ldots, \alpha_{n}\right) \in N^{n}$.

1.2. We assume that we are given differential operators

$$
A(z, D)=\sum_{k q_{0}+\langle\alpha, q\rangle \leqq \mu} a_{k \alpha} z^{k} D^{\alpha} \quad\left(k \in N, \alpha \in N^{n}\right)
$$


and

$$
B_{j}(z, D)=\sum_{k q_{0}+\langle\alpha, q\rangle \leqq \mu_{j}} b_{j k \alpha} z^{k} D^{\alpha}, \quad j=1, \ldots, \varkappa,
$$

depending on the parameter $z \in Z \subset C$, where the coefficients $a_{k x}$ and $b_{j k x}$ are complex constants, $\mu_{j} \geqq 0$ is the order of $B_{j}(z, D)$, and $\varkappa$ is a positive integer. The corresponding principal symbols are

and

$$
A^{0}(z, \xi)=\sum_{k q_{0}+\langle\alpha, q\rangle=\mu} a_{k \alpha} z^{k} \xi^{\alpha}
$$

$$
B_{j}^{0}(z, \xi)=\sum_{k q_{0}+\langle\alpha, q\rangle=\mu_{j}} b_{j k \alpha} z^{k} \xi^{\alpha}, \quad j=1, \ldots, x .
$$

The parameter set $Z$ will be a sector $Z=Z\left(\omega_{1}, \omega_{2}\right) \subset \boldsymbol{C}, \omega_{1} \leqq \omega_{2}$,

$$
Z\left(\omega_{1}, \omega_{2}\right)=\left\{z \in \boldsymbol{C} \mid \omega_{1} \leqq \arg z \leqq \omega_{2}\right\} .
$$

1.3. The operator system

$$
\left(A(z, D), B_{1}(z, D), \ldots, B_{\varkappa}(z, D)\right), \quad 1 \leqq \chi \leqq m_{n},
$$

is said to be parametrically quasi-elliptic in $Z$ if

(i) $A^{0}(z, \xi) \neq 0$ for all $z \in Z$ and all $\xi \in \boldsymbol{R}^{n}$ such that $h(z, \xi)>0$;

(ii) for every $z \in Z$ and every $\xi^{\prime} \in \boldsymbol{R}^{n-1}$ with $h\left(z, \xi^{\prime}\right)>0$, the polynomial $A^{0}\left(z, \xi^{\prime}, \zeta\right)$ in (the complex variable) $\zeta$ has exactly $\chi$ roots $\zeta_{j}^{+}\left(z, \xi^{\prime}\right), j=1, \ldots, \varkappa$, with $\operatorname{Im} \zeta_{j}^{+}\left(z, \xi^{\prime}\right)>0$;

(iii) for every $z \in Z$ and every $\xi^{\prime} \in \boldsymbol{R}^{n-1}$ with $h\left(z, \xi^{\prime}\right)>0$, the polynomials $B_{j}^{0}\left(z, \xi^{\prime}, \zeta\right)$ in $\zeta, j=1, \ldots, \varkappa$, are linearly independent modulo

(See [6].)

$$
A^{+}\left(z, \zeta^{\prime}, \zeta\right)=\sum_{j=1}^{\varkappa}\left(\zeta-\zeta_{j}^{+}\left(z, \zeta^{\prime}\right)\right) .
$$

\section{Quasi-parabolic problems}

2.1. We shall employ the notation

$$
\begin{aligned}
& \boldsymbol{R}_{+}^{n}=\left\{x=\left(x^{\prime}, x_{n}\right) \in \boldsymbol{R}^{n} \mid x_{n}>0\right\}, \\
& Q=R_{+} \times R_{+}^{n} \subset \boldsymbol{R}_{t} \times \boldsymbol{R}_{x}^{n}, \\
& \Sigma=\boldsymbol{R}_{+} \times \boldsymbol{R}^{n-1} \subset \boldsymbol{R}_{t} \times \boldsymbol{R}_{x^{\prime}}^{n-1} .
\end{aligned}
$$

Let $\partial_{t}$ stand for the operator $\partial / \partial t$, and let $\gamma_{t}$ and $\gamma_{n}$ denote the trace operators with respect to $t$ and $x_{n}$, respectively, i.e.

$$
\gamma_{t} u=\left.u\right|_{t=0}, \quad \gamma_{n} u=\left.u\right|_{x_{n}=0} .
$$

2.2. Problem $(Q P)$. Let us consider the operators (see 1.2)

$$
A\left(\partial_{t}, D\right)=\sum_{k q_{0}+\langle\alpha, q\rangle \leqq \mu} a_{k \alpha} \partial_{t}^{k} D^{\alpha}
$$


and

$$
B_{j}\left(\partial_{t}, D\right)=\sum_{k q_{0}+\langle\alpha, q\rangle \leqq \mu_{j}} b_{j k \alpha} \partial_{t}^{k} D^{\alpha}, \quad j=1, \ldots, \varkappa .
$$

The formal setting of the initial-boundary value problem (QP) which we shall study is now as follows:

$$
\begin{array}{ll}
A\left(\partial_{t}, D\right) u=f & \text { in } \quad Q, \\
\gamma_{t} \partial_{t}^{k} u=\varphi_{k} & \text { on } \boldsymbol{R}_{+}^{n}, \quad k=0, \ldots, m_{0}-1, \\
\gamma_{n} B_{j}\left(\partial_{t}, D\right) u=g_{j} & \text { on } \Sigma, j=1, \ldots, \varkappa .
\end{array}
$$

We shall come later on to the realization of this problem, the actual object of study, by fixing the function spaces to which the data $f, \varphi_{k}, g_{j}$, and the solution $u$ are to belong.

2.3. We say that the operator system

$$
\left(A\left(\partial_{t}, D\right),\left(\gamma_{t} \partial_{t}^{k}\right)_{k},\left(\gamma_{n} B_{j}\left(\partial_{t}, D\right)\right)_{j}\right)
$$

is quasi-parabolic and that (QP) is a quasi-parabolic problem if the operator system

$$
\left(A(z, D), B_{1}(z, D), \ldots, B_{\varkappa}(z, D)\right)
$$

is parametrically quasi-elliptic in the sector $Z=Z(-\pi / 2, \pi / 2)$.

\section{SOME FUNCTION SPACES}

\section{3. $H^{s}$-spaces}

3.1. If $X$ is a (complex) normed space, its norm will always be denoted by $\|\cdot\|_{X} \cdot$

The normed space of bounded (linear) operators of a normed space $X$ into another normed space $Y$ is denoted by $L(X ; Y)$.

If $X, Y$, and $Z$ are three normed spaces such that $X \subset Z$ and $Y \subset Z$ algebraically and topologically, we shall equip the space $V=X \cap Y$ with the norm

$$
\|u\|_{V}=\left(\|u\|_{X}^{2}+\|u\|_{Y}^{2}\right)^{1 / 2}
$$

Note that if $X$ and $Y$ are Hilbert spaces, so is $X \cap Y$.

3.2. Let $X$ be a Hilbert space, and let $\Omega$ be an open set in $\boldsymbol{R}^{k}$.

Let $C_{c}^{\infty}(\Omega ; X)$ denote the space of $C^{\infty}$-functions $u: \Omega \rightarrow X$ having compact support supp $u \subset \Omega$, and let $C_{c}^{\infty}(\bar{\Omega} ; X)$ be the space of restrictions to $\bar{\Omega}$ of $C_{c}^{\infty}\left(\boldsymbol{R}^{k} ; X\right)$-functions, where $\bar{\Omega}$ means the closure of $\Omega$.

Let $\mathscr{D}^{\prime}(\Omega ; X)$ be the space of $X$-valued distributions in $\Omega, \mathscr{S}^{\prime}\left(\boldsymbol{R}^{k} ; X\right)$ the space of tempered distributions, and $L^{2}(\Omega ; X)$ the Lebesgue space. 
Suppose now that $\boldsymbol{R}^{k}=\boldsymbol{R}_{y}^{k}$ is $\boldsymbol{R}_{t}, \boldsymbol{R}_{x_{n}}, \boldsymbol{R}_{x^{\prime}}^{n-1}$ or $\boldsymbol{R}_{x}^{n}$, and set

$$
K(\eta)=(1+\langle\eta\rangle)^{1 / 2}
$$

where $\eta$ is the dual vector of $y$. For $s \geqq 0$ we then recall the definition of the anisotropic Sobolev space $H^{s}(\Omega ; X)$ of order $s$ :

First,

with the norm

$$
H^{s}\left(\boldsymbol{R}_{\boldsymbol{y}}^{k} ; X\right)=\left\{u \in \mathscr{S}^{\prime}\left(\boldsymbol{R}_{y}^{k} ; X\right) \mid K(\eta)^{s} \mathscr{F}_{y} u \in L^{2}\left(\boldsymbol{R}_{\eta}^{k} ; X\right)\right\}
$$

$$
\|u\|_{H^{s}\left(\boldsymbol{R}^{k} ; X\right)}=\left\|K(\eta)^{s} \mathscr{F}_{y} u\right\|_{L^{2}\left(\boldsymbol{R}^{k} ; X\right)}
$$

where $\mathscr{F}_{y} u$ is the Fourier transform of $u$,

and then

$$
\left(\mathscr{F}_{y} u\right)(\eta)=\int e^{-i\langle y, \eta\rangle} u(y) d y,
$$

with the norm

$$
H^{s}(\Omega ; X)=\left\{r_{\Omega} U \mid U \in H^{s}\left(\boldsymbol{R}^{k} ; X\right)\right\}
$$

$$
\|u\|_{H^{s}(\Omega ; X)}=\inf \left\{\|U\|_{H^{s}\left(R^{k} ; X\right)} \mid U \in H^{s}\left(\boldsymbol{R}^{k} ; X\right), r_{\Omega} U=u\right\} ;
$$

here $r_{\Omega}$ is the operator restricting to $\Omega$ functions (distributions) defined in $R^{k}$.

Note that $H^{0}(\Omega ; X)=L^{2}(\Omega ; X)$.

When $X=C$, the symbol $C$ will be omitted.

For trace theorems and other basic results for $H^{s}(\Omega ; X)$-spaces we refer to the works of L. Hörmander, J. L. Lions and E. Magenes, L. R. Volevič and B. P. Panejah, and other authors cited in Lions-Magenes [4], p. 105, and also to L. N. Slobodeckiǐ [8] and M. Troisi [9].

3.3. For any $z \in \boldsymbol{C}$ the Hilbert space $H_{z}^{s}\left(\boldsymbol{R}_{+}^{n}\right)$ is defined to be the space $H^{s}\left(\boldsymbol{R}_{+}^{n}\right)$ equipped with the norm

$$
\|u\|_{H_{z}^{s}\left(R_{+}^{n}\right)}=\left(\|u\|_{H^{s}\left(R_{+}^{n}\right)}^{2}+\langle z\rangle^{2 s}\|u\|_{H^{0}\left(R_{+}^{n}\right)}^{2}\right)^{1 / 2}
$$

the definition of the space $H_{z}^{s}\left(\boldsymbol{R}^{n-1}\right)$ is analogous. (See [6].)

3.4. For $\boldsymbol{R}_{+}=\boldsymbol{R}_{t,+}$ let $H_{0}^{s}\left(\boldsymbol{R}_{+} ; X\right)$ be the closure in $H^{s}\left(\boldsymbol{R}_{+} ; X\right)$ of $C_{c}^{\infty}\left(\boldsymbol{R}_{+} ; X\right)$, and if $s=k q_{0}+q_{0} / 2$ with $k \in \boldsymbol{N}$, set

$$
H_{00}^{s}\left(\boldsymbol{R}_{+} ; X\right)=\left\{u \in H_{0}^{s}\left(\boldsymbol{R}_{+} ; X\right) \mid t^{-1 / 2} \partial_{t}^{k} u \in L^{2}\left(\boldsymbol{R}_{+} ; X\right)\right\}
$$

equipped with the norm whose square is given by

$$
\|u\|_{H^{s}\left(R_{+} ; X\right)}^{2}+\left\|t^{-1 / 2} \partial_{t}^{k} u\right\|_{L^{2}\left(R_{+} ; X\right)}^{2}
$$

(cf. Lions-Magenes [4], Chap. I, § 11). We define then the Hilbert space $H_{(0)}^{s}\left(\boldsymbol{R}_{+} ; X\right)$ as the space $H_{0}^{s}\left(\boldsymbol{R}_{+} ; X\right)$ if $s \not \equiv q_{0} / 2 \bmod q_{0}$ and as the space $H_{00}^{s}\left(\boldsymbol{R}_{+} ; X\right)$ if $s \equiv q_{0} / 2 \bmod q_{0}$. 
3.5. For $\boldsymbol{R}_{+}=\boldsymbol{R}_{t,+}$ and for any $\varrho \geqq 0$ we define the Hilbert space $H^{s}\left(\boldsymbol{R}_{+} ; \varrho ; X\right)$ by

$$
H^{s}\left(\boldsymbol{R}_{+} ; \varrho ; X\right)=\left\{u \in \mathscr{D}^{\prime}\left(\boldsymbol{R}_{+} ; X\right) \mid e^{-\varrho t} u \in H^{s}\left(\boldsymbol{R}_{+} ; X\right)\right\}
$$

with the norm

$$
\|u\|_{H^{s}\left(R_{+} ; \varrho ; X\right)}=\left\|e^{-\ell t} u\right\|_{H^{s}\left(R_{+} ; X\right)} .
$$

The space $H_{(0)}^{s}\left(\boldsymbol{R}_{+} ; \varrho ; X\right)$ and its norm are introduced similarly.

\section{4. $H^{(s)}$-spaces}

4.1. For $s \geqq 0$ and $r \geqq 0$, define (see 3.1 )

$$
\begin{aligned}
& H^{s, r}(Q)=H^{s}\left(\boldsymbol{R}_{+} ; H^{0}\left(\boldsymbol{R}_{+}^{n}\right)\right) \cap H^{0}\left(\boldsymbol{R}_{+} ; H^{r}\left(\boldsymbol{R}_{+}^{n}\right)\right), \\
& H_{(0)}^{s, r}(Q)=H_{(0)}^{s}\left(\boldsymbol{R}_{+} ; H^{0}\left(\boldsymbol{R}_{+}^{n}\right)\right) \cap H^{0}\left(\boldsymbol{R}_{+} ; H^{r}\left(\boldsymbol{R}_{+}^{n}\right)\right),
\end{aligned}
$$

and analogously the spaces $H^{s, r}(\Sigma)$ and $H_{(0)}^{s, r}(\Sigma)$.

We set, for any $\varrho \geqq 0$,

$$
H^{s, r}(Q ; \varrho)=H^{s}\left(\boldsymbol{R}_{+} ; \varrho ; H^{0}\left(\boldsymbol{R}_{+}^{n}\right)\right) \cap H^{0}\left(\boldsymbol{R}_{+} ; \varrho ; H^{r}\left(\boldsymbol{R}_{+}^{n}\right)\right)
$$

or, equivalently,

with the norm

$$
H^{s, r}(Q ; \varrho)=\left\{u \in \mathscr{D}^{\prime}(Q) \mid e^{-\varrho t} u \in H^{s, r}(Q)\right\}
$$

$$
\|u\|_{H^{s, r}(Q ; \varrho)}=\left\|e^{-\varrho t} u\right\|_{H^{s, r}(Q)} .
$$

In similar way one introduces the space $H^{s, r}(\Sigma ; \varrho)$ and further the spaces $H_{(0)}^{s, r}(Q ; \varrho)$ and $H_{(0)}^{s, r}(\Sigma ; \varrho)$.

The following three propositions can be derived, for example, from the results of Slobodeckii [8], or directly proved by methods closely related to those used in Grisvard [2]; see also Lions-Magenes [5], pp. 9-12. Without further mention, we assume that $\varrho \geqq 0$ is given.

4.2. Proposition. Suppose $s>0, r>0$, and $k \in N, \alpha \in N^{n}$ such that

$$
\frac{k q_{0}}{s}+\frac{\langle\alpha, q\rangle}{r} \leqq 1 \text {. }
$$

Then $\partial_{t}^{k} D^{\alpha}$ is a continuous operator

with

$$
H^{s, r}(Q ; \varrho) \rightarrow H^{s_{k}, r_{\alpha}}(Q ; \varrho)
$$

$$
\frac{s_{k}}{s}=\frac{r_{\alpha}}{r}=1-\left(\frac{k q_{0}}{s}+\frac{\langle\alpha, q\rangle}{r}\right)
$$

(the operator norm of $\partial_{t}^{k} D^{\alpha}$ being less or equal to $P_{k}(\varrho)$, where $P_{k}$ is a positive polynomial of degree $k$ in $\varrho$ ).

An analogous result holds for the space $H^{s, r}(\Sigma ; \varrho)$. 
4.3. Proposition. Suppose $s>0$ and $r>q_{n} / 2$. If $j \in N$ with $r-j q_{n}-q_{n} / 2>0$, then $\gamma_{n} D_{n}^{j}$ is a continuous surjective operator

$$
H^{s, r}(Q ; \varrho) \rightarrow H^{s_{j}, r_{j}}(\Sigma ; \varrho)
$$

$\left(C_{c}^{\infty}(\bar{Q})\right.$ is dense in $\left.H^{s, r}(Q ; \varrho)\right)$, where

$$
\frac{s_{j}}{s}=\frac{r_{j}}{r}=1-\frac{j q_{n}+q_{n} / 2}{r} .
$$

4.4. Proposition. Suppose $s>q_{0} / 2$ and $r>0$. If $k \in N$ such that $s-k q_{0}-$ $q_{0} / 2>0$, then $\gamma_{t} \partial_{t}^{k}$ is a continuous surjective operator

and

$$
H^{s, r}(Q ; \varrho) \rightarrow H^{p_{k}}\left(\boldsymbol{R}_{+}^{n}\right)
$$

where

$$
H^{s, r}(\Sigma ; \varrho) \rightarrow H^{p_{k}}\left(\boldsymbol{R}^{n-1}\right),
$$

$$
\frac{p_{k}}{r}=1-\frac{k q_{0}+q_{0} / 2}{s} .
$$

4.5. In the sequel we shall employ the $H^{s, r}$-spaces in the case $s=r$. It is therefore convenient to introduce the notation $H^{(s)}=H^{s, s}$.

The following two results can be obtained from Propositions 4.3 and 4.4, respectively, by the open mapping theorem, for example.

4.6. Proposition. Let $\varrho \geqq 0$. If $s>q_{n} / 2$, then the mapping

is a surjective operator

$$
\gamma_{n}^{s}=\left(\gamma_{n} D_{n}^{j}\right)_{j q_{n}<s-q_{n} / 2}
$$

$$
H^{(s)}(Q ; \varrho) \rightarrow \prod_{j} H^{\left(s-j q_{n}-q_{n} / 2\right)}(\Sigma ; \varrho),
$$

and it has a continuous linear right inverse $\left(\gamma_{n}^{s}\right)_{R}^{-1}$.

4.7. Proposition. Let $\varrho \geqq 0$. If $s>q_{0} / 2$, then the mapping

is a surjective operator

$$
\gamma_{t}^{s}=\left(\gamma_{t} \partial_{t}^{k}\right)_{k q_{0}<s-q_{0} / 2}
$$

$$
H^{(s)}(Q ; \varrho) \rightarrow \prod_{k} H^{s-k q_{0}-q_{0} / 2}\left(\boldsymbol{R}_{+}^{n}\right),
$$

and it has a continuous linear right inverse $\left(\gamma_{t}^{s}\right)_{R}^{-1}$.

4.8. Let $X$ and $Y$ be two (separable) Hilbert spaces forming an interpolation couple $\{X, Y\}$, that is, $X \subset Y$ algebraically and topologically such that $X$ is dense in $Y$, and denote the corresponding intermediate spaces by $[X, Y]_{\theta}, 0<\theta<1$ (see Lions-Magenes [4], Chap. I). We recall two basic results (see [4], pp. 27-28):

First, if $\{\mathscr{X}, \mathscr{Y}\}$ is another interpolation couple and if

$$
\Lambda \in L(X ; \mathscr{X}) \cap L(Y ; \mathscr{Y}),
$$


then the interpolation theorem says that

$$
\Lambda \in L\left([X, Y]_{\theta} ;[\mathscr{X}, \mathscr{Y}]_{\theta}\right), \quad 0<\theta<1 .
$$

Second, reiteration holds: For $0<\alpha<\beta<1$,

$$
\left[[X, Y]_{\alpha},[X, Y]_{\beta}\right]_{\theta}=[X, Y]_{(1-\theta) \alpha+\theta \beta}, \quad 0<\theta<1,
$$

with equivalent norms.

We are now in a position to state the following result, obtained by methods similar to those used in [4], Chap. I, $\S \$ 11,13$ (cf. also [5], Chap. 4, § 2).

4.9. Proposition. Suppose $s>r \geqq 0$, and let $\Omega$ stand for $Q$ or $\Sigma$. Then

and

$$
\left[H^{(s)}(\Omega), H^{(r)}(\Omega)\right]_{\theta}=H^{((1-\theta) s+\theta r)}(\Omega)
$$

$$
\left[H_{(0)}^{(s)}(\Omega), H_{(0)}^{(r)}(\Omega)\right]_{\theta}=H_{(0)}^{((1-\theta) s+\theta r)}(\Omega)
$$

(with equivalent norms) for every $\theta, 0<\theta<1$.

\section{5. $\mathscr{H}^{s}$-spaces}

Throughout this section, let $X$ denote a Hilbert space, and suppose $\varrho>0$ and $s \geqq 0$.

5.1. For $\boldsymbol{R}=\boldsymbol{R}_{t}$, we define the Hilbert space $H_{+}^{s}(\boldsymbol{R} ; \varrho ; X)$ by

$$
H_{+}^{s}(\boldsymbol{R} ; \varrho ; X)=\left\{u \in \mathscr{D}^{\prime}(\boldsymbol{R} ; X) \mid \operatorname{supp} u \subset \overline{\boldsymbol{R}}_{+}, e^{-\varrho t} u \in H^{s}(\boldsymbol{R} ; X)\right\}
$$

with the norm

$$
\|u\|_{H_{+}^{s}(R ; \varrho ; X)}=\left\|e^{-\varrho t} u\right\|_{H^{s}(R ; X)} .
$$

This space is not essentially new. In fact, we have

5.2. Lemma. The mapping

$$
\begin{aligned}
& I_{0}: H_{(0)}^{s}\left(\boldsymbol{R}_{+} ; \varrho ; X\right) \rightarrow H_{+}^{s}(\boldsymbol{R} ; \varrho ; X), \\
& I_{0} u=u^{\sim} \quad\left(u^{\sim}=\text { extension of } u \text { by zero for } t<0\right),
\end{aligned}
$$

is an isomorphism with inverse

$$
\begin{aligned}
& I_{+}: H_{+}^{s}(\boldsymbol{R} ; \varrho ; X) \rightarrow H_{(0)}^{s}\left(\boldsymbol{R}_{+} ; \varrho ; X\right), \\
& I_{+} v=r_{+} v \quad\left(r_{+}=\text {restriction operator } r_{R_{+}}\right) .
\end{aligned}
$$

Proof. We are going to show that $I_{0}$ is a continuous bijective linear mapping. Then, since $H_{(0)}^{s}\left(\boldsymbol{R}_{+} ; \varrho ; X\right)$ and $H_{+}^{s}(\boldsymbol{R} ; \varrho ; X)$ are complete spaces, the open mapping theorem implies that $I_{0}$ is an isomorphism.

5.2.1. From the definition of $H_{(0)}^{s}\left(\boldsymbol{R}_{+} ; \varrho ; X\right)$ it easily follows that $I_{0}$ maps $H_{(0)}^{s}\left(\boldsymbol{R}_{+} ; \varrho ; X\right)$ into $H_{+}^{s}(\boldsymbol{R} ; \varrho ; X)$. 
5.2.2. Let us suppose that $v \in H_{+}^{s}(\boldsymbol{R} ; \varrho ; X)$. Then $V=e^{-\varrho t} v \in H^{s}(\boldsymbol{R} ; X)$ satisfies $\operatorname{supp} V \subset \overline{\boldsymbol{R}}_{+}$, and if we set $u=I_{+} v=r_{+} v$, we have

$$
U=e^{-e t} u=r_{+} V \in H^{s}\left(\boldsymbol{R}_{+} ; X\right) .
$$

Now, it is not difficult to see that

$$
U \in H_{0}^{s}\left(\boldsymbol{R}_{+} ; X\right)
$$

Therefore, at least,

$$
I_{+} v=u \in H_{0}^{s}\left(\boldsymbol{R}_{+} ; \varrho ; X\right) .
$$

In the case $s=k q_{0}+q_{0} / 2, k \in N$, we must continue. To do this, set $V_{k}=\partial_{t}^{k} V$. Then

$$
V_{k} \in H^{q_{0} / 2}(\boldsymbol{R} ; X) \text { and } \operatorname{supp} V_{k} \subset \bar{R}_{+} .
$$

Furthermore,

$$
W_{k}=r_{+} V_{k} \in H^{q_{0} / 2}\left(\boldsymbol{R}_{+} ; X\right), \quad W_{k}=\partial_{t}^{k} U .
$$

By (1), we first conclude (cf. [4], p. 52) that

Hence, in particular,

$$
\int_{0}^{\infty} \tau^{-2} d \tau \int\left\|V_{k}(t+\tau)-V_{k}(t)\right\|_{X}^{2} d t<\infty
$$

$$
\int_{0}^{\infty} \tau^{-2} d \tau \int_{0}^{\tau}\left\|W_{k}(t)\right\|_{X}^{2} d t<\infty .
$$

From this it follows (cf. Hewitt-Stromberg [3], § 18) that

$$
\int_{0}^{\infty} t^{-1}\left\|\partial_{t}^{k} U\right\|_{X}^{2} d t=\int_{0}^{\infty} \tau^{-1}\left\|W_{k}(\tau)\right\|_{X}^{2} d \tau<\infty .
$$

Thus we obtain $U \in H_{(0)}^{s}\left(\boldsymbol{R}_{+} ; X\right)$, so that

$$
I_{+} v=u \in H_{(0)}^{s}\left(\boldsymbol{R}_{+} ; \varrho ; X\right) .
$$

5.2.3. If $u \in H_{(0)}^{s}\left(\boldsymbol{R}_{+} ; \varrho ; X\right)$, we obviously have $I_{+} I_{0} u=r_{+} u^{\sim}=u$. Since supp $v \subset \overline{\boldsymbol{R}}_{+}$for $v \in H_{+}^{s}(\boldsymbol{R} ; \varrho ; X)$, we have also $I_{0} I_{+} v=\left(r_{+} v\right)^{\sim}=v$. Accordingly, $I_{+}=I_{0}^{-1}$.

Finally, the definition of $H_{(0)}^{s}\left(\boldsymbol{R}_{+} ; X\right)$ implies that $I_{0}$ is continuous.

5.3. We now introduce the normed space $\mathscr{H}^{s}\left(\boldsymbol{C}_{e} ; X\right)$, where

$$
\boldsymbol{C}_{\varrho}=\{z \in \boldsymbol{C} \mid \operatorname{Re} z>\varrho\},
$$

as follows: We say that $U \in \mathscr{H}^{s}\left(\boldsymbol{C}_{\varrho} ; X\right)$ if

(i) $U: \boldsymbol{C}_{\boldsymbol{Q}} \rightarrow X$ is holomorphic,

(ii) $\sup _{\sigma>\varrho} \int_{-\infty}^{\infty}\langle\sigma+i \tau\rangle^{2 s}\|U(\sigma+i \tau)\|_{X}^{2} d \tau=\sup _{\sigma>\varrho} \frac{1}{i} \int_{\sigma-i \infty}^{\sigma+i \infty}\langle z\rangle^{2 s}\|U(z)\|_{X}^{2} d z<\infty$,

and equip $\mathscr{H}^{s}\left(\boldsymbol{C}_{\varrho} ; X\right)$ with the norm whose square, for $U \in \mathscr{H}^{s}\left(\boldsymbol{C}_{\varrho} ; X\right)$, is given by the expression of (ii). 
From Theorem 7.1 of Agranovič-Višik [1] (the proof of this result for $X$ valued functions is essentially the same) it follows that the Laplace transformation $\mathscr{L}$,

$$
(\mathscr{L} u)(z)=\int_{0}^{\infty} e^{-z t} u(t) d t,
$$

is an isomorphism of $H_{+}^{s}(\boldsymbol{R} ; \varrho ; X)$ onto $\mathscr{H}^{s}\left(\boldsymbol{C}_{\varrho} ; X\right)$. In view of Lemma 5.2 we thus have (an adapted Paley-Wiener theorem)

5.4. Proposition. The Laplace transformation $\mathscr{L}\left(\right.$ or $\left.\mathscr{L}_{\circ} I_{0}\right)$ is an isomorphism of $H_{(0)}^{s}\left(\boldsymbol{R}_{+} ; \varrho ; X\right)$ onto $\mathscr{H}^{s}\left(\boldsymbol{C}_{\varrho} ; X\right)$ with inverse $\mathscr{L}^{-1}$.

5.5. Corollary. $\mathscr{H}^{s}\left(\boldsymbol{C}_{\boldsymbol{e}} ; X\right)$ is complete.

5.6. Corollary. If $U \in \mathscr{H}^{s}\left(C_{e} ; X\right)$, then

$$
\|U\|_{\mathscr{H}^{s}\left(C_{e} ; X\right)}^{2}=\int\langle\varrho+i \tau\rangle^{2 s}\|U(\varrho+i \tau)\|_{X}^{2} d \tau
$$

with $U(\varrho+i \tau)=\mathscr{F}_{t}\left(e^{--\varrho t} \mathscr{L}^{-1} U\right)^{\sim}$ (see Lemma 5.2).

Proof. First, take $U \in \mathscr{H}^{0}\left(\boldsymbol{C}_{\varrho} ; X\right)$ and set $u=\mathscr{L}^{-1} U$. Then

$$
U(\varrho+i \tau)=\mathscr{F}_{t}\left(e^{-\varrho t} u\right)^{\sim} \in L^{2}\left(\boldsymbol{R}_{\tau} ; X\right) .
$$

By the Parseval formula we now obtain

$$
\begin{aligned}
\|U\|_{\mathscr{H}^{0}\left(C_{\varrho} ; X\right)}^{2} & =\sup _{\sigma>\varrho} \int\|U(\sigma+i \tau)\|_{X}^{2} d \tau=2 \pi \int_{0}^{\infty} e^{-2 \varrho t}\|u\|_{X}^{2} d t \\
& =\int\|U(\varrho+i \tau)\|_{X}^{2} d \tau .
\end{aligned}
$$

Next, suppose $U \in \mathscr{H}^{s}\left(\boldsymbol{C}_{\varrho} ; X\right)$ with $s>0$. Then $z^{s / q_{0}} U \in \mathscr{H}^{0}\left(\boldsymbol{C}_{\varrho} ; X\right)$, so that

$$
\|U\|_{\mathscr{H}^{s}\left(C_{\varrho} ; X\right)}^{2}=\left\|z^{s / q_{0}} U\right\|_{\mathscr{H}^{0}\left(C_{\varrho} ; X\right)}^{2}=\int\langle\varrho+i \tau\rangle^{2 s}\|U(\varrho+i \tau)\|_{X}^{2} d \tau .
$$

In the following statement we use the notation and concepts introduced in 3.1 and 4.8 .

5.7. Proposition. Let $Y$ be a Hilbert space such that $X$ and $Y$ form an interpolation couple $\{X, Y\}$, and suppose $s>0$. Then for every $r, 0 \leqq r \leqq s$, the mapping

is a continuous operator

$$
U \mapsto z^{r / q_{0}} U
$$

$$
\mathscr{H}^{0}\left(\boldsymbol{C}_{\varrho} ; X\right) \cap \mathscr{H}^{s}\left(\boldsymbol{C}_{\varrho} ; Y\right) \rightarrow \mathscr{H}^{0}\left(\boldsymbol{C}_{\varrho} ;[X, Y]_{r / s}\right) \cap \mathscr{H}^{s-r}\left(\boldsymbol{C}_{\varrho} ; Y\right) .
$$

Proof. The idea of the proof is as follows: Let $\Lambda$ be a self-adjoint positive operator in $Y$ such that $[X, Y]_{\theta}$ is the domain of $\Lambda^{1-\theta}, 0 \leqq \theta \leqq 1$. Making use of the diagonalization of $\Lambda$ by a unitary operator of $Y$ onto a direct hilbertian integral with respect to a positive Radon measure, we are then able to transform 
the assertion into a scalar inequality. Since the proof is not far from the one given in [4], pp. 14-18, for intermediate derivatives of $L^{2}$-functions, we omit the details.

5.8. If $k \in N$ such that $k q_{0} \leqq s$, then it follows from $3.5,4.8$ and an analogue of Proposition 4.9 that, for any $u \in H_{(0)}^{s}\left(\boldsymbol{R}_{+} ; \varrho ; X\right)$,

$$
\partial_{t}^{k} u \in H_{(0)}^{s-k q_{0}}\left(\boldsymbol{R}_{+} ; \varrho ; X\right) .
$$

Thus we have, by Proposition 5.4,

Moreover,

$$
\mathscr{L}\left(\partial_{t}^{k} u\right) \in \mathscr{H}^{s-k q_{0}}\left(C_{\varrho} ; X\right) .
$$

(see Schwartz [7], p. 246).

$$
\mathscr{L}\left(\partial_{t}^{k} u\right)=z^{k} \mathscr{L} u
$$

\section{6. $\mathscr{H}^{(s)}$-spaces}

Let us assume $\varrho>0$.

6.1. For every $s \geqq 0$, we define (see 3.1 )

$$
\mathscr{H}^{(s)}\left(\boldsymbol{C}_{\boldsymbol{e}}, \Omega\right)=\mathscr{H}^{s}\left(\boldsymbol{C}_{\boldsymbol{e}} ; H^{0}(\Omega)\right) \cap \mathscr{H}^{0}\left(\boldsymbol{C}_{\boldsymbol{e}} ; H^{s}(\Omega)\right),
$$

where $\Omega$ stands for $\boldsymbol{R}_{+}^{n}$ or $\boldsymbol{R}^{n-1}$. Notice that Corollary 5.6 (see also 3.3) yields

$$
\|U\|_{\mathscr{H}}^{2(s)}\left(C_{e}, \Omega\right)=\int\|U(\varrho+i \tau)\|_{H_{Q}^{s}+i \tau}^{2}(\Omega) d \tau .
$$

As a consequence of Proposition 5.4 we obtain the following result.

6.2. Proposition. For any $s \geqq 0$, the Laplace transformation $\mathscr{L}$ is an isomorphism of $H_{(0)}^{(s)}\left(\boldsymbol{R}_{+} \times \Omega\right.$; @) onto $\mathscr{H}^{(s)}\left(\boldsymbol{C}_{\varrho}, \Omega\right)$, where $\Omega$ is $\boldsymbol{R}_{+}^{n}$ or $\boldsymbol{R}^{n-1}$.

Also the next four lemmas will be needed in the sequel; the proofs are given in 6.7.

6.3. Lemma. Let $\Lambda\left(\partial_{t}, D\right)$ be a differential operator of order $\lambda$, and suppose $s \geqq \lambda$. Then $\Lambda(z, D)$ is a continuous operator of $\mathscr{H}^{(s)}\left(\boldsymbol{C}_{\varrho}, \boldsymbol{R}_{+}^{n}\right)$ into $\mathscr{H}^{(s-\lambda)}\left(\boldsymbol{C}_{\varrho}, \boldsymbol{R}_{+}^{n}\right)$.

6.4. Lemma. Let $\Lambda\left(\partial_{t}, D\right)$ and $s$ be as in Lemma 6.3, and suppose furthermore $s>\lambda$ if $\lambda \equiv q_{0} / 2 \bmod q_{0}$. Then

for all $u \in H_{(0)}^{(s)}(Q ; \varrho)$.

$$
\mathscr{L} \Lambda\left(\partial_{t}, D\right) u=\Lambda(z, D) \mathscr{L} u
$$

6.5. Lemma. If $s>q_{n} / 2$, the trace operator $\gamma_{n}$ is defined and continuous on $\mathscr{H}^{(s)}\left(\boldsymbol{C}_{\varrho}, \boldsymbol{R}_{+}^{n}\right)$ with values in $\mathscr{H}^{\left(s-q_{n} / 2\right)}\left(\boldsymbol{C}_{\varrho}, \boldsymbol{R}^{n-1}\right)$.

6.6. Lemma. If $s>q_{n} / 2$, then

for all $u \in H_{(0)}^{(s)}(Q ; \varrho)$.

$$
\mathscr{L} \gamma_{n} u=\gamma_{n} \mathscr{L} u
$$


6.7. The proofs of Lemmas 6.3 to 6.6.

6.7.1. Proof of Lemma 6.3. Rewrite $\Lambda(z, D)$ in the form

$$
\Lambda(z, D)=\sum_{k q_{0} \leqq \lambda} \Lambda^{(k)}(D) z^{k},
$$

where $\Lambda^{(k)}(D)$ is a differential operator of order $\leqq \lambda-k q_{0}$. By Proposition 5.7 we see that $U \mapsto z^{k} U$ is a continuous operator

$$
\mathscr{H}^{(s)}\left(\boldsymbol{C}_{\varrho}, \boldsymbol{R}_{+}^{n}\right) \rightarrow \mathscr{H}^{\left(s-k q_{0}\right)}\left(\boldsymbol{C}_{\varrho}, \boldsymbol{R}_{+}^{n}\right) .
$$

Since $\Lambda^{(k)}(D)$ is obviously a continuous operator

$$
\mathscr{H}^{0}\left(\boldsymbol{C}_{\varrho} ; H^{s-k q_{0}}\left(\boldsymbol{R}_{+}^{n}\right)\right) \rightarrow \mathscr{H}^{0}\left(\boldsymbol{C}_{\varrho} ; H^{s-\lambda}\left(\boldsymbol{R}_{+}^{n}\right)\right),
$$

we obtain the assertion by 6.1 and by Lemma 2.2 of [6].

6.7.2. Proof of Lemma 6.4. First, we have

6.7.3. Lemma. For any $s \geqq 0$, the set

$$
C_{c, 0}^{\infty}(\bar{Q})=\left\{u \in C_{c}^{\infty}(\bar{Q}) \mid \operatorname{supp} u \subset \boldsymbol{R}_{+} \times \overline{\mathbf{R}}_{+}^{n}\right\}
$$

is dense in $H_{(0)}^{(s)}(Q)$ and in $H_{(0)}^{(s)}(Q ; \varrho)$.

To see this, it suffices to use basic properties of $H_{(0)}^{(s)}$ and intermediate spaces.

Next, by 4.8, 4.9, and Lemma 6.7.3 we have

6.7.4. Lemma. If the hypotheses of Lemma 6.4 are satisfied, then

and

$$
\Lambda\left(\partial_{t}, D\right) \in L\left(H_{(0)}^{(s)}(Q) ; H_{(0)}^{(s-\lambda)}(Q)\right)
$$

$$
\Lambda\left(\partial_{t}, D\right) \in L\left(H_{(0)}^{(s)}(Q ; \varrho) ; H_{(0)}^{(s-\lambda)}(Q ; \varrho)\right) .
$$

Now we see, by Lemma 6.7.4 and Proposition 6.2, that $\mathscr{L} \Lambda\left(\partial_{t}, D\right)$ is a continuous operator of $H_{(0)}^{(s)}(Q ; \varrho)$ into $\mathscr{H}^{(s-\lambda)}\left(\boldsymbol{C}_{\varrho}, \boldsymbol{R}_{+}^{n}\right)$, and so is $\Lambda\left(\partial_{t}, D\right) \mathscr{L}$, by virtue of Proposition 6.2 and Lemma 6.3. Since these two operators coincide on the dense set $C_{c, 0}^{\infty}(\bar{Q})$, they do so on $H_{(0)}^{(s)}(Q ; \varrho)$.

6.7.5. Proof of Lemma 6.5. For any $U \in \mathscr{H}^{(s)}\left(\boldsymbol{C}_{\varrho}, \boldsymbol{R}_{+}^{n}\right)$, we have

so that

$$
U(z) \in H^{s}\left(\boldsymbol{R}_{+}^{n}\right), \quad z \in \boldsymbol{C}_{\varrho},
$$

and

$$
\gamma_{n} U(z) \in H^{s-q_{n} / 2}\left(\boldsymbol{R}^{n-1}\right)
$$

$$
\left\|\gamma_{n} U(z)\right\|_{H^{s-q_{n} / 2\left(R^{n-1}\right)}} \leqq C\|U(z)\|_{H^{s}\left(R_{+}^{n}\right)}
$$

by the trace theorem (see Slobodeckir [8] and Troisi [9], for example). It therefore follows that $\gamma_{n} U$ is holomorphic in $\boldsymbol{C}_{e}$ with

$$
\frac{d}{d z}\left(\gamma_{n} U\right)=\gamma_{n} \frac{d U}{d z}
$$


In view of Lemma 1.9 of [6], we then conclude that

and that

$$
\gamma_{n} U \in \mathscr{H}^{\left(s-q_{n} / 2\right)}\left(\boldsymbol{C}_{\varrho}, \boldsymbol{R}^{n-1}\right)
$$

$$
\left\|\gamma_{n} U\right\|_{\mathscr{H}^{\left(s-q_{n} / 2\right)}\left(C_{\varrho}, R^{n-1)}\right.} \leqq C\|U\|_{\mathscr{H}^{(s)}\left(C_{\varrho}, R_{+}^{n}\right)}
$$

6.7.6. Proof of Lemma 6.6. Using Proposition 4.9 and an analogue of Theorem 4.2 of [4], p. 24, one can prove

6.7.7. Lemma. If $s>q_{n} / 2$, then $\gamma_{n}$ is a continuous operator

(see Proposition 4.3).

$$
H_{(0)}^{(s)}(Q ; \varrho) \rightarrow H_{(0)}^{\left(s-q_{n} / 2\right)}(\Sigma ; \varrho)
$$

Now we see that $\mathscr{L} \gamma_{n}$ is a continuous operator of $H_{(\mathbf{0})}^{(s)}(Q ; \varrho)$ into $\mathscr{H}^{\left(s-q_{n} / 2\right)}\left(\boldsymbol{C}_{\varrho}, \boldsymbol{R}^{n-1}\right)$, by Proposition 6.2 and Lemma 6.7.7, and that so is $\gamma_{n} \mathscr{L}$, by Proposition 6.2 and Lemma 6.5. Since $\mathscr{L} \gamma_{n}$ and $\gamma_{n} \mathscr{L}$ coincide on $C_{c, 0}^{\infty}(\bar{Q})$, the result follows.

6.8. Suppose that we are given the operators $B_{j}\left(\partial_{t}, D\right)$ of order $\mu_{j}$, $j=1, \ldots, x$, as in 2.2. Let $s \in \boldsymbol{R}$ be such that

$$
s>\mu_{j}+q_{n} / 2, \quad j=1, \ldots, \varkappa .
$$

Let us now introduce the space

$$
H_{(0), B}^{(s)}(Q ; \varrho)=\left\{u \in H_{(0)}^{(s)}(Q ; \varrho) \mid \gamma_{n} B_{j}\left(\partial_{t}, D\right) u=0, j=1, \ldots, \varkappa\right\},
$$

a closed subspace of $H_{(0)}^{(s)}(Q ; \varrho)$ (see Lemmas 6.7.4 and 6.7.7), and the space

$$
\mathscr{H}_{B}^{(s)}\left(\boldsymbol{C}_{\varrho}, \boldsymbol{R}_{+}^{n}\right)=\left\{U \in \mathscr{H}^{(s)}\left(\boldsymbol{C}_{\varrho}, \boldsymbol{R}_{+}^{n}\right) \mid \gamma_{n} B_{j}(z, D) U=0, j=1, \ldots, \varkappa\right\},
$$

a closed subspace of $\mathscr{H}^{(s)}\left(\boldsymbol{C}_{\varrho}, \boldsymbol{R}_{+}^{n}\right)$ (see Lemmas 6.3 and 6.5). Then we have

6.9. Proposition. $\mathscr{L}\left(H_{(0), B}^{(s)}(Q ; \varrho)\right)=\mathscr{H}_{B}^{(s)}\left(\boldsymbol{C}_{\varrho}, \boldsymbol{R}_{+}^{n}\right)$.

Proof. For any $u \in H_{(0), B}^{(s)}(Q ; \varrho)$, we have, by Proposition 6.2,

$$
\mathscr{L} u \in \mathscr{H}^{(s)}\left(\boldsymbol{C}_{\varrho}, \boldsymbol{R}_{+}^{n}\right),
$$

and furthermore, by Lemmas 6.4, 6.5, and 6.6,

so that

$$
\gamma_{n} B_{j}(z, D) \mathscr{L} u=\mathscr{L}\left(\gamma_{n} B_{j}\left(\partial_{t}, D\right) u\right)=0,
$$

$$
\mathscr{L} u \in \mathscr{H}_{B}^{(s)}\left(\boldsymbol{C}_{\varrho}, \boldsymbol{R}_{+}^{n}\right) .
$$

On the other hand, if $U \in \mathscr{H}_{B}^{(s)}\left(\boldsymbol{C}_{\varrho}, \boldsymbol{R}_{+}^{n}\right)$, then a similar reasoning shows that

$$
\mathscr{L}^{-1} U \in H_{(0), B}^{(s)}(Q ; \varrho) .
$$




\section{INITIAL AND BOUNDARY VALUES}

\section{Initial values}

7.1. Let $A_{t}(\partial, D)$ be the differential operator given in 2.2 , and write

$$
A\left(\partial_{t}, D\right)=\sum_{k=0}^{m_{0}} A^{(k)}(D) \partial_{t}^{k}
$$

with

$$
A^{(k)}(D)=\sum_{\langle\alpha, q\rangle \leqq \mu-k q_{0}} a_{k \alpha} D^{\alpha}, \quad k=0, \ldots, m_{0} .
$$

Assuming now that $a_{m_{0}, 0}=1$, we have

$$
\partial_{t}^{m_{0}}=A\left(\partial_{t}, D\right)-\sum_{k=0}^{m_{0}-1} A^{(k)}(D) \partial_{t}^{k} .
$$

Note that the preceding hypothesis is essentially satisfied if, for example, $A(z, D)$ is parametrically quasi-elliptic, i.e., satisfies condition 1.3 (i).

Given $u \in H^{(s)}(Q ; \varrho)$ with $s \geqq \mu, \varrho \geqq 0$, we set

and

$$
f=A\left(\partial_{t}, D\right) u \in H^{(s-\mu)}(Q ; \varrho)
$$

moreover, let

$$
\Phi_{k}=\gamma_{t} \partial_{t}^{k} u \in H^{s-k q_{0}-q_{0} / 2}\left(\boldsymbol{R}_{+}^{n}\right) \quad \text { for } \quad k q_{0}<s-q_{0} / 2
$$

By (1), we see that now

$$
\varphi_{k}=\Phi_{k} \text { when } \quad 0 \leqq k \leqq m_{0}-1 .
$$

for every $r \in N$ with $r q_{0}<s-\mu-q_{0} / 2$.

$$
\Phi_{m_{0}+r}=\gamma_{t} \partial_{t}^{r} f-\sum_{k=0}^{m_{0}-1} A^{(k)}(D) \Phi_{r+k}
$$

Let us next introduce the operators

$$
\begin{array}{ll}
S_{-r}=0, & r=1,2, \ldots, \\
S_{0}=-\mathrm{Id} & (\mathrm{Id}=\text { identity }) \\
S_{r}=-\sum_{k=0}^{m_{0}-1} A^{(k)}(D) S_{r-m_{0}+k}, & r=1,2, \ldots,
\end{array}
$$

and

$$
\begin{array}{ll}
T_{-r}=0, & r=1,2, \ldots, \\
T_{0}=\gamma_{t}, & \\
T_{r}=\gamma_{t} \partial_{t}^{r}-\sum_{k=0}^{m_{0}-1} A^{(k)}(D) T_{r-m_{0}+k}, & r=1,2, \ldots
\end{array}
$$

Then for any $s \geqq 0, S_{r}$ is a continuous operator of $H^{s}\left(\boldsymbol{R}_{+}^{n}\right)$ into $H^{s-r q_{0}}\left(\boldsymbol{R}_{+}^{n}\right)$ when $r q_{0} \leqq s$, and $T_{r}$ is a continuous operator

$$
H^{s}(Q ; \varrho) \rightarrow H^{s-r q_{0}-q_{0} / 2}\left(\boldsymbol{R}_{+}^{n}\right) \quad \text { when } \quad r q_{0}<s-q_{0} / 2 .
$$



values.

With our previous notation, we now have the following result about initial

7.2. Theorem. Suppose that the coefficient of $\partial_{t}^{m_{0}}$ in the operator $A\left(\partial_{t}, D\right)$ is equal to one, and that $s \geqq \mu, \varrho \geqq 0$. Then, given any $u \in H^{(s)}(Q ; \varrho)$, the relation

$$
\Phi_{m_{0}+r}=T_{r} f+\sum_{v=0}^{m_{0}-1} S_{r-v} \sum_{k=0}^{m_{0}-1-v} A^{(k)}(D) \varphi_{v+k}
$$

holds whenever $0 \leqq r q_{0}<s-\mu-q_{0} / 2$.

Proof. The assertion can be proved by induction on $r$; however, we omit the details.

\section{Initial and boundary values. The spaces $G^{s}(\varrho), F^{s}(\varrho)$, and $E^{s}(\varrho)$}

We assume throughout this section that the operators $B_{j}\left(\partial_{t}, D\right), j=1, \ldots, \varkappa$, are given as in 2.2 , and write

where

$$
B_{j}\left(\partial_{t}, D\right)=\sum_{k q_{0} \leqq \mu_{j}} B_{j}^{(k)}(D) \partial_{t}^{k}
$$

$$
B_{j}^{(k)}(D)=\sum_{\langle\alpha, q\rangle \leqq \mu_{j}-k q_{0}} b_{j k \alpha} D^{\alpha}, \quad 0 \leqq k q_{0} \leqq \mu_{j} .
$$

Suppose also that $\varrho \geqq 0$, and that

$$
s>\mu_{j}+q_{n} / 2 \text { for every } j=1, \ldots, \varkappa .
$$

8.1. Let us now introduce the Hilbert space

$$
G^{s}(\varrho)=G^{s}(\varrho, B)=\prod_{k} H^{s-k q_{0}-q_{0} / 2}\left(\boldsymbol{R}_{+}^{n}\right) \times \prod_{j} H^{\left(s-\mu_{j}-q_{n} / 2\right)}(\Sigma ; \varrho)
$$

where $k \in N$ with $k q_{0}<s-q_{0} / 2$ and $j=1, \ldots, \varkappa$. Then the mapping

$$
\Psi=\left(\left(\gamma_{t} \partial_{t}^{k}\right)_{k},\left(\gamma_{n} B_{j}\left(\partial_{t}, D\right)\right)_{j}\right)
$$

(with $k$ and $j$ as above) is a continuous operator of $H^{(s)}(Q ; \varrho)$ into $G^{s}(\varrho)$.

Thus we have, for any $u \in H^{(s)}(Q ; \varrho)$,

if we use the notation

$$
\Psi u=\left(\left(\Phi_{k}\right)_{k},\left(g_{j}\right)_{j}\right) \in G^{s}(\varrho)
$$

$$
\begin{array}{ll}
\Phi_{k}=\gamma_{t} \partial_{t}^{k} u, & k q_{0}<s-q_{0} / 2, \\
g_{j}=\gamma_{n} B_{j}\left(\partial_{t}, D\right) u, & j=1, \ldots, \varkappa .
\end{array}
$$

Let us also set

$$
\delta=\frac{1}{2}\left(q_{0}+q_{n}\right) .
$$


The initial and boundary values now satisfy the following local compatibility relations (cf. Grisvard [2]):

8.2. Theorem. Suppose $\mu_{j}+v q_{0}<s-\delta, v \in N$. Then

$$
\gamma_{t} \partial_{t}^{v} g_{j}=\gamma_{n} \sum_{k q_{0} \leqq \mu_{j}} B_{j}^{(k)}(D) \Phi_{v+k}
$$

for every $u \in H^{(s)}(Q ; \varrho), \Psi u=\left(\left(\Phi_{k}\right)_{k},\left(g_{j}\right)_{j}\right)$.

Proof. Employing Propositions 4.2, 4.3, and 4.4 (in addition to the usual trace theorems), we see that the mappings

and

$$
u \mapsto \gamma_{t} \partial_{t}^{v} g_{j}
$$

$$
u \mapsto \gamma_{n} \sum_{k q_{0} \leqq \mu_{j}} B_{j}^{(k)}(D) \Phi_{v+k}
$$

are both continuous operators of $H^{(s)}(Q ; \varrho)$ into $H^{s-\mu_{j}-v q_{0}-\delta}\left(\boldsymbol{R}^{n-1}\right)$. Since they coincide on the dense subset $C_{c}^{\infty}(\bar{Q})$, the statement follows.

The preceding result is completed by the following global compatibility relation (cf. Grisvard [2]):

8.3. Theorem. Suppose $s-\mu_{j} \equiv \delta \bmod q_{0}$, say, $s-\mu_{j}-\delta=v q_{0}$ with $v \in \boldsymbol{N}$. Then

$$
\begin{aligned}
& \int_{0}^{\infty} \int\left|\sum_{k q_{0} \leqq \mu_{j}}\left(B_{j}^{(k)}(D) \Phi_{v+k}\right)\left(x^{\prime}, \sigma^{s / q_{0}}\right)-\exp \left(-\varrho \sigma^{s / q_{n}}\right)\left(\partial_{t}^{v} g_{j}\right)\left(\sigma^{s / q_{n}, x^{\prime}}\right)\right|^{2} d x^{\prime} \frac{d \sigma}{\sigma} \\
& \leqq C\|u\|_{H^{(s)}(Q ; \varrho)}^{2}
\end{aligned}
$$

for all $u \in H^{(s)}(Q ; \varrho), \Psi u=\left(\left(\Phi_{k}\right)_{k},\left(g_{j}\right)_{j}\right)$.

Proof. For the proof we need the next lemma which can be proved as a result of P. Grisvard (see Théorème 5.1 in [2] or Theorem 2.2 in Lions-Magenes [5], p. 13).

8.3.1. Lemma. Let $X$ be a Hilbert space. Suppose $\boldsymbol{R}_{+} \times \boldsymbol{R}_{+} \subset \boldsymbol{R}_{t} \times \boldsymbol{R}_{x_{n}}$, and define (cf. 4.1)

$$
H^{(\delta)}\left(\boldsymbol{R}_{+} \times \boldsymbol{R}_{+} ; \varrho ; X\right)=H^{\delta}\left(\boldsymbol{R}_{+} ; \varrho ; H^{0}\left(\boldsymbol{R}_{+} ; X\right)\right) \cap H^{0}\left(\boldsymbol{R}_{+} ; \varrho ; H^{\delta}\left(\boldsymbol{R}_{+} ; X\right)\right) .
$$

Given any $u \in H^{(\delta)}\left(\boldsymbol{R}_{+} \times \boldsymbol{R}_{+} ; \varrho ; X\right)$, set

Then

$$
\begin{aligned}
& f=\gamma_{t} u \in H^{q_{n} / 2}\left(\boldsymbol{R}_{x_{n},+} ; X\right), \\
& g=\gamma_{n} u \in H^{q_{0} / 2}\left(\boldsymbol{R}_{t,+} ; \varrho ; X\right) .
\end{aligned}
$$

$$
\int_{0}^{\infty}\left\|f\left(\sigma^{\delta / q_{0}}\right)-\exp \left(-\varrho \sigma^{\delta / q_{n}}\right) g\left(\sigma^{\delta / q_{n}}\right)\right\|_{X}^{2} \frac{d \sigma}{\sigma} \leqq C\|u\|_{H^{(\delta)}\left(R_{+} \times R_{+} ; \varrho ; X\right)}^{2}
$$

for all $u \in H^{(\delta)}\left(\boldsymbol{R}_{+} \times \boldsymbol{R}_{+} ; \varrho ; X\right)$.

8.3.2. We shall prove a formally more general result. 
Let $\Lambda=\Lambda\left(\partial_{t}, D_{x^{\prime}}\right)$ be a differential operator of order $\lambda \leqq s-\mu_{j}-\delta$, and write

and similarly, for brevity,

$$
\Lambda=\sum_{v q_{0} \leqq \lambda} \Lambda^{(v)} \partial_{t}^{v} \quad \text { with } \quad \Lambda^{(v)}=\Lambda^{(v)}\left(D_{x^{\prime}}\right)
$$

$$
B_{j}=B_{j}\left(\partial_{t}, D\right), \quad B_{j}^{(k)}=B_{j}^{(k)}(D) .
$$

Let now $u \in H^{(s)}(Q ; \varrho)$. Then, by Proposition 4.2,

$$
\Lambda B_{j} u \in H^{(\delta)}(Q ; \varrho) \subset H^{(\delta)}\left(\boldsymbol{R}_{+} \times \boldsymbol{R}_{+} ; \varrho ; H^{0}\left(\boldsymbol{R}^{n-1}\right)\right),
$$

where the inclusion is both algebraic and topological. Thus it follows from Lemma 8.3.1 that

where

$$
\begin{aligned}
& \int_{0}^{\infty} \int \mid\left(\Lambda B_{j} u\right)\left(0, x^{\prime}, \sigma^{\delta / q_{0}}\right)-\exp \left(-\varrho \sigma^{\delta / q_{n}}\right)\left(\Lambda B_{j} u\right)\left(\sigma^{\left.\delta / q_{n}, x^{\prime}, 0\right)\left.\right|_{i} ^{2} d x^{\prime}} \frac{d \sigma}{\sigma}\right. \\
& \leqq C\|u\|_{H^{(s)}(Q ; \varrho)}^{2}
\end{aligned}
$$

and

$$
\left(\Lambda B_{j} u\right)(0, x)=\gamma_{t}\left(\Lambda B_{j} u\right)(t, x)=\sum_{v q_{0} \leqq \lambda} \sum_{k q_{0} \leqq \mu_{j}}\left(\Lambda^{(v)} B_{j}^{(k)} \Phi_{v+k}\right)(x)
$$

$$
\left(\Lambda B_{j} u\right)\left(t, x^{\prime}, 0\right)=\gamma_{n}\left(\Lambda B_{j} u\right)(t, x)=\left(\Lambda g_{j}\right)\left(t, x^{\prime}\right)
$$

(cf. the reasoning in the proof of Theorem 8.2). Thus we finally obtain, by substituting $\sigma^{s}$ for $\sigma^{\delta}$,

$$
\begin{gathered}
\int_{0}^{\infty} \int\left|\sum_{v q_{0} \leqq \lambda} \sum_{k q_{0} \leqq \mu_{j}}\left(\Lambda^{(v)} B_{j}^{(k)} \Phi_{v+k}\right)\left(x^{\prime}, \sigma^{s / q_{0}}\right)-\exp \left(-\varrho \sigma^{s / q_{n}}\right)\left(\Lambda g_{j}\right)\left(\sigma^{s / q_{n}}, x^{\prime}\right)\right|^{2} d x^{\prime} \frac{d \sigma}{\sigma} \\
\leqq C\|u\|_{H^{(s)}(Q ; \varrho)}^{2},
\end{gathered}
$$

which at once implies the desired inequality; notice that the implication holds also vice versa.

8.4. Let $F^{s}(\varrho)$ denote the space of such $\left(\left(\Phi_{k}\right)_{k},\left(g_{j}\right)_{j}\right) \in G^{s}(\varrho)$ that the conditions (LCR) and (GCR) are satisfied:

(LCR) If $\mu_{j}+v q_{0}<s-\delta$ with $v \in N$, then

$$
\gamma_{t} \partial_{t}^{v} g_{j}=\gamma_{n} \sum_{k q_{0} \leqq \mu_{j}} B_{j}^{(k)}(D) \Phi_{v+k} .
$$

(GCR) If $\mu_{j}+v q_{0}=s-\delta$ with $v \in N$, then

$$
\int_{0}^{\infty} \int\left|\sum_{k q_{0} \leqq \mu_{j}}\left(B_{j}^{(k)}(D) \Phi_{v+k}\right)\left(x^{\prime}, \sigma^{s / q_{0}}\right)-\exp \left(-\varrho \sigma^{s / q_{n}}\right)\left(\partial_{t}^{v} g_{j}\right)\left(\sigma^{s / q_{n}}, x^{\prime}\right)\right|^{2} d x^{\prime} \frac{d \sigma}{\sigma}<\infty \text {. }
$$

If we now set, for every $\left(\left(\Phi_{k}\right)_{k},\left(g_{j}\right)_{j}\right) \in F^{s}(\varrho)$,

$$
\begin{aligned}
\left\|\left(\left(\Phi_{k}\right)_{k},\left(g_{j}\right)_{j}\right)\right\|_{F^{s}(\varrho)}^{2} & =\left.\left\|\left(\left(\Phi_{k}\right)_{k},\left(g_{j}\right)_{j}\right)\right\|_{j}^{2}\right|_{G^{s}(\varrho)} \\
& +\left.\sum_{\mu_{j}+v q_{0}=s-\delta} \int_{0}^{\infty} \int\right|_{k u_{0} \leqq \mu_{j}} \sum_{j}\left(B_{j}^{(k)}(D) \Phi_{v+k}\right)\left(x^{\prime}, \sigma^{s / q_{0}}\right) \\
& -\left.\exp \left(-\varrho \sigma^{s / q_{n}}\right)\left(\partial_{t}^{v} g_{j}\right)\left(\sigma^{s / q_{n},}, x^{\prime}\right)\right|^{2} d x^{\prime} \frac{d \sigma}{\sigma},
\end{aligned}
$$


then $F^{s}(\varrho)$ is a Hilbert space with the norm so defined. One can show this by using the completeness of $G^{s}(\varrho)$ and the fact that

$$
\begin{aligned}
& H^{q_{n} / 2}\left(\boldsymbol{R}_{+}^{n}\right) \subset L^{2}\left(\boldsymbol{R}_{+} ; H^{0}\left(\boldsymbol{R}^{n-1}\right)\right), \\
& H^{q_{0} / 2}(\Sigma ; \varrho) \subset L^{2}\left(\boldsymbol{R}_{+} ; \varrho ; H^{0}\left(\boldsymbol{R}^{n-1}\right)\right) ;
\end{aligned}
$$

we omit the (somewhat tedious) details.

8.5. To conclude this section, define

$$
E^{s}(\varrho)=\Psi\left(H^{(s)}(Q ; \varrho)\right)
$$

The space $E^{s}(\varrho)$ will be regarded not only as a vector subspace of $G^{s}(\varrho)$ but also as a normed subspace of $F^{s}(\varrho)$, which is possible because of Theorems 8.2 and 8.3. Consequently, the operator

$$
\Psi: H^{(s)}(Q ; \varrho) \rightarrow E^{s}(\varrho)
$$

is surjective and, by Theorem 8.3, continuous. Furthermore, if the kernel of $\Psi$ is denoted (for informative and uniform notation) by $H_{\Psi}^{(s)}(Q ; \varrho)$, and its orthogonal complement by $H_{\Psi}^{(s)}(Q ; \varrho)^{\perp}$, we see that $\Psi$ induces in a natural way a continuous bijective operator

$$
\Psi_{0}: H_{\Psi}^{(s)}(Q ; \varrho)^{\perp} \rightarrow E^{s}(\varrho)
$$

Note that $H_{(0), B}^{(s)}(Q ; \varrho) \subset H_{\Psi}^{(s)}(Q ; \varrho) ;$ in particular, if $s \neq \equiv q_{0} / 2 \bmod q_{0}$, then $H_{(0), B}^{(s)}(Q ; \varrho)=H_{\Psi}^{(s)}(Q ; \varrho)$.

\section{QUASI-PARABOLIC PROBLEMS}

We return now to the quasi-parabolic problem (QP) of Section 2.

In the following sections we shall study the existence, uniqueness, and a priori estimates of solutions to Problem (QP).

Let us therefore assume that the operators $A\left(\partial_{t}, D\right)$ and $B_{i}\left(\partial_{t}, D\right)$, $j=1, \ldots, x$, are given as in 2.2 , and that the operator system

$$
\left(A\left(\partial_{t}, D\right),\left(\gamma_{t} \partial_{t}^{k}\right)_{k},\left(\gamma_{n} B_{j}\left(\partial_{t}, D\right)\right)_{j}\right)
$$

is quasi-parabolic in the sense of 2.3. We assume also that the leading coefficient $a_{m_{0}, 0}$ of $A\left(\partial_{t}, D\right)$ is equal to one (see 7.1).

For brevity, we shall use the notation

$$
\begin{aligned}
& R=R\left(\mu, \mu_{j}\right)=\left\{s \in \boldsymbol{R} \mid s \geqq \mu, s>\max \left\{\mu_{j}\right\}+q_{n} / 2\right\}, \\
& R_{q_{0}}=\left\{s \in R \mid s \not \equiv q_{0} / 2 \bmod q_{0}\right\} .
\end{aligned}
$$


9. A priori estimates in $\mathscr{H}^{(s)}\left(\boldsymbol{C}_{\varrho}, \boldsymbol{R}_{+}^{n}\right)$ and $H_{(0)}^{(s)}(Q ; \varrho)$

In this section we derive some basic inequalities from the results of [6].

9.1. Theorem. If $s \in R$, then for $\varrho>0$ large enough the estimate

$$
\|U\|_{\mathscr{H}^{(s)}\left(C_{e}, R_{+}^{n}\right)} \leqq C\left(\|A(z, D) U\|_{\mathscr{H}^{(s-\mu)}\left(C_{e}, R_{+}^{n}\right)}+\sum_{j}\left\|\gamma_{n} B_{j}(z, D) U\right\|_{\mathscr{H}^{\left(s-\mu_{j}-q_{n} / 2\right)}\left(C_{\varrho}, R^{n-1}\right)}\right)
$$

holds for all $U \in \mathscr{H}^{(s)}\left(\boldsymbol{C}_{\varrho}, \boldsymbol{R}_{+}^{n}\right)$; in particular,

$$
\|U\|_{\mathscr{H}^{(s)}\left(C_{\varrho}, R_{+}^{n}\right)} \leqq C\|A(z, D) U\|_{\mathscr{H}^{(s-\mu)}\left(C_{\varrho}, R_{+}^{n}\right)}
$$

for all $U \in \mathscr{H}_{B}^{(s)}\left(\boldsymbol{C}_{\varrho}, \boldsymbol{R}_{+}^{n}\right)$.

Proof. We first observe that everything in the statement is well-defined by virtue of Lemmas 6.3 and 6.5. By Theorem 4.3 of [6] we can now find $\varrho>0$ such that, for any $U \in \mathscr{H}^{(s)}\left(\boldsymbol{C}_{\varrho}, \boldsymbol{R}_{+}^{n}\right)$ and for all $z \in \boldsymbol{C}_{\varrho}$,

$$
\|U(z)\|_{H_{z}^{s}\left(R_{+}^{n}\right)}^{2} \leqq C\left(\|A(z, D) U(z)\|_{H_{z}^{s-\mu}\left(R_{+}^{n}\right)}^{2}+\sum_{j}\left\|\gamma_{n} B_{j}(z, D) U(z)\right\|_{H_{z}^{s-\mu_{j}-q_{n} / 2}\left(R^{n-1}\right)}^{2}\right) .
$$

But by 6.1 , this yields at once what we wanted.

9.2. Theorem. Suppose $s \in R$, and let $\varrho>0$ be as in Theorem 9.1. Then

$$
\|u\|_{H_{(0)}^{(s)}(Q ; \varrho)} \leqq C\left(\left\|A\left(\partial_{t}, D\right) u\right\|_{H_{(0)}^{(s-\mu)}(Q ; \varrho)}+\sum_{j}\left\|\gamma_{n} B_{j}\left(\partial_{t}, D\right) u\right\|_{H_{(0)}^{\left(s-\mu_{j}-q_{n} / 2\right)}(\Sigma ; \varrho)}\right)
$$

for all $u \in H_{(0)}^{(s)}(Q ; \varrho)$.

Proof. For $u \in H_{(\mathbf{0})}^{(s)}(Q ; \varrho)$ we have, by Proposition 6.2,

$$
U=\mathscr{L} u \in \mathscr{H}^{(s)}\left(\boldsymbol{C}_{\varrho}, \boldsymbol{R}_{+}^{n}\right),
$$

and furthermore, by Lemmas 6.4 and 6.6,

$$
\begin{aligned}
A(z, D) U & =\mathscr{L}\left(A\left(\partial_{t}, D\right) u\right), \\
\gamma_{n} B_{j}(z, D) U & =\mathscr{L}\left(\gamma_{n} B_{j}\left(\partial_{t}, D\right) u\right) .
\end{aligned}
$$

Applying now Theorem 9.1 and again Proposition 6.2, we obtain the asserted inequality.

In the same manner one verifies the following theorem, using, in addition, Proposition 6.9.

9.3. Theorem. Suppose $s \in R$, and let $\varrho>0$ be as in Theorem 9.1. Then

$$
\|u\|_{H_{(0)}^{(s)}(Q ; \varrho)} \leqq C\left\|A\left(\partial_{t}, D\right) u\right\|_{H_{(0)}^{(s-\mu)}(Q ; \varrho)}
$$

for all $u \in H_{(0), B}^{(s)}(Q ; \varrho)$.

9.4. Note that the converses of the estimates in Theorems 9.1 to 9.3 hold for any $\varrho>0$. 


\section{Existence and uniqueness of solutions for homogeneous initial and boundary values}

10.1. Theorem. Let $s \in R$ be given. If $\varrho>0$ is sufficiently large, then for every $F \in \mathscr{H}^{(s-\mu)}\left(\boldsymbol{C}_{\varrho}, \boldsymbol{R}_{+}^{n}\right)$ the problem

$$
A(z, D) U=F
$$

has a unique solution $U \in \mathscr{H}_{B}^{(s)}\left(C_{\varrho}, R_{+}^{n}\right)$.

Proof. First of all, we choose $\varrho>0$ such that we are free to apply Theorems 4.3 and 4.4 of [6].

10.1.1. Suppose $F \in \mathscr{H}^{(s-\mu)}\left(\boldsymbol{C}_{\varrho}, \boldsymbol{R}_{+}^{n}\right)$. Then $F(z) \in H^{s-\mu}\left(\boldsymbol{R}_{+}^{n}\right)$ for every $z \in \boldsymbol{C}_{\underline{Q}}$. Thus it follows from Theorem 4.4 of [6] that the problem

$$
\begin{aligned}
& A(z, D) u=F(z), \\
& \gamma_{n} B_{j}(z, D) u=0, \quad j=1, \ldots, x,
\end{aligned}
$$

has a unique solution $u=U(z) \in H^{s}\left(\boldsymbol{R}_{+}^{n}\right)$.

We are now going to show that the mapping $z \mapsto U(z)$ is holomorphic.

10.1.2. The mapping

is holomorphic, and

$$
z \mapsto A(z, D): C \rightarrow L\left(H^{s}\left(\boldsymbol{R}_{+}^{n}\right) ; H^{s-\mu}\left(\boldsymbol{R}_{+}^{n}\right)\right)
$$

$$
\frac{d}{d z} A(z, D)=A^{\prime}(z, D)=\sum_{0<k q_{0} \leqq \mu} k z^{k-1} A^{(k)}(D) ;
$$

likewise, for each $j=1, \ldots, \chi$,

$$
\frac{d}{d z} B_{j}(z, D)=B_{j}^{\prime}(z, D)=\sum_{0<k q_{0} \leqq \mu_{j}} k z^{k-1} B_{j}^{(k)}(D)
$$

(of course, $B_{j}^{\prime}=0$ if $B_{j}=B_{j}^{(0)}$ ).

Thus we have, for every $z \in C_{\ell}$,

moreover,

$$
\begin{aligned}
& A^{\prime}(z, D) U(z) \in H^{s-\mu}\left(\boldsymbol{R}_{+}^{n}\right), \\
& \gamma_{n} B_{j}^{\prime}(z, D) U(z) \in H^{s-\mu_{j}-q_{n} / 2}\left(\boldsymbol{R}^{n-1}\right) ;
\end{aligned}
$$

$$
F^{\prime}(z)=\frac{d F}{d z}(z) \in H^{s-\mu}\left(\boldsymbol{R}_{+}^{n}\right) .
$$

Applying Theorem 4.4 of [6], we may therefore conclude that the problem

$$
\begin{aligned}
& A(z, D) v=F^{\prime}(z)-A^{\prime}(z, D) U(z), \\
& \gamma_{n} B_{j}(z, D) v=-\gamma_{n} B_{j}^{\prime}(z, D) U(z), \quad j=1, \ldots, \varkappa,
\end{aligned}
$$

has a unique solution $v=V(z) \in H^{s}\left(\boldsymbol{R}_{+}^{n}\right)$. 
10.1.3. Let now $z \in C_{\varrho}$ be fixed, and suppose $\Delta z \in C, \Delta z \neq 0$, such that $z+\Delta z \in C_{\varrho}$. Let us write

If we then set

$$
\begin{aligned}
& \Delta F(z)=F(z+\Delta z)-F(z), \\
& \Delta U(z)=U(z+\Delta z)-U(z), \\
& \Delta A(z, D)=A(z+\Delta z, D)-A(z, D), \\
& \Delta B_{j}(z, D)=B_{j}(z+\Delta z, D)-B_{j}(z, D) .
\end{aligned}
$$

$$
W(z, \Delta z)=\frac{\Delta U(z)}{\Delta z}-V(z) \in H^{s}\left(\boldsymbol{R}_{+}^{n}\right),
$$

we have, by (1) and (3),

$$
\begin{aligned}
A(z, D) W(z, \Delta z) & =\left(\frac{\Delta F(z)}{\Delta z}-F^{\prime}(z)\right) \\
& -\left(\frac{\Delta A(z, D)}{\Delta z}-A^{\prime}(z, D)\right) U(z+\Delta z)-A^{\prime}(z, D) \Delta U(z),
\end{aligned}
$$

and similarly, by (2) and (4),

$$
\gamma_{n} B_{j}(z, D) W(z, \Delta z)=-\gamma_{n}\left(\frac{\Delta B_{j}(z, D)}{\Delta z}-B_{j}^{\prime}(z, D)\right) U(z+\Delta z)-\gamma_{n} B_{j}^{\prime}(z, D) \Delta U(z) .
$$

By virtue of Theorem 4.3 of [6] we thus obtain

$$
\text { (5) } \begin{aligned}
\|W(z, \Delta z)\|_{H^{s}\left(R_{+}^{n}\right)} \leqq C & \left\|\frac{\Delta F(z)}{\Delta z}-F^{\prime}(z)\right\|_{H^{s-\mu}\left(R_{+}^{n}\right)} \\
& +\left\|\left(\frac{\Delta A(z, D)}{\Delta z}-A^{\prime}(z, D)\right) U(z+\Delta z)\right\|_{H^{s-\mu}\left(R_{+}^{n}\right)} \\
& +\left\|A^{\prime}(z, D) \Delta U(z)\right\|_{H^{s-\mu}\left(R_{+}^{n}\right)} \\
& +\sum_{j}\left\|\gamma_{n}\left(\frac{\Delta B_{j}(z, D)}{\Delta z}-B_{j}^{\prime}(z, D)\right) U(z+\Delta z)\right\|_{H^{s-\mu_{j}-q_{n} / 2}\left(R^{n-1}\right)} \\
& \left.+\sum_{j}\left\|\gamma_{n} B_{j}^{\prime}(z, D) \Delta U(z)\right\|_{H^{s-\mu_{j}-q_{n} / 2}\left(R^{n-1}\right)}\right) .
\end{aligned}
$$

To handle the right side of (5), we now observe, by (1), (2), and Theorem 4.3 of [6], that there exist $M>0$ and $\eta>0$ such that

Since (1) and (2) yield

$$
\|U(z+\Delta z)\|_{H^{s}\left(R_{+}^{n}\right)} \leqq M \quad \text { if } \quad|\Delta z| \leqq \eta .
$$

$$
\begin{aligned}
& A(z, D) \Delta U(z)=\Delta F(z)-\Delta A(z, D) U(z+\Delta z), \\
& \gamma_{n} B_{j}(z, D) \Delta U(z)=-\gamma_{n} \Delta B_{j}(z, D) U(z+\Delta z), \quad j=1, \ldots, \chi,
\end{aligned}
$$

we also obtain, again by using Theorem 4.3 of [6],

$$
\|\Delta U(z)\|_{H^{s}\left(R_{+}^{n}\right)} \rightarrow 0 \text { as } \quad \Delta z \rightarrow 0 .
$$


But then it follows from (5) that

$$
\left\|\frac{\Delta U(z)}{\Delta z}-V(z)\right\|_{H^{s}\left(R_{+}^{n}\right)} \rightarrow 0 \quad \text { as } \quad \Delta z \rightarrow 0 .
$$

Thus we have proved that the mapping

is holomorphic.

$$
z \mapsto U(z): C_{\varrho} \rightarrow H^{s}\left(\boldsymbol{R}_{+}^{n}\right)
$$

10.1.4. It is now obvious (see 6.1) that $U \in \mathscr{H}^{(s)}\left(\boldsymbol{C}_{\varrho}, \boldsymbol{R}_{+}^{n}\right)$ since

$$
\|U(z)\|_{H_{z}^{s}\left(R_{+}^{n}\right)} \leqq C\|F(z)\|_{H_{z}^{s-\mu}\left(R_{+}^{n}\right)}
$$

by virtue of Theorem 4.3 of [6]. Hence we have, by (2),

$$
U \in \mathscr{H}_{B}^{s}\left(C_{\varrho}, R_{+}^{n}\right) .
$$

Therefore, by (1), this $U$ is a solution. Since the uniqueness follows at once from Theorem 9.1, the proof is complete.

10.2. Theorem. Suppose $s \in R$, and let $\varrho>0$ be as in Theorem 10.1. Then for every $f \in H_{(0)}^{(s-\mu)}(Q ; \varrho)$ the problem

$$
A\left(\partial_{t}, D\right) u=f
$$

has a unique solution $u \in H_{(0), B}^{(s)}(Q ; \varrho)$.

Proof. Suppose $f \in H_{(0)}^{(s-\mu)}(Q ; \varrho)$, and take

$$
F=\mathscr{L} f \in \mathscr{H}^{(s-\mu)}\left(\boldsymbol{C}_{\varrho}, \boldsymbol{R}_{+}^{n}\right)
$$

by means of Proposition 6.2. By virtue of Theorem 10.1 we then know that the equation

$$
A(z, D) U=F
$$

has a solution $U \in \mathscr{H}_{\boldsymbol{B}}^{(\boldsymbol{s})}\left(\boldsymbol{C}_{\varrho}, \boldsymbol{R}_{+}^{n}\right)$. If we now apply Propositions 6.2 and 6.9 , we see that

$$
u=\mathscr{L}^{-1} U \in H_{(0), B}^{(s)}(Q ; \varrho) .
$$

Thus it follows from (1), by Proposition 6.2 and Lemma 6.4, that

$$
A\left(\partial_{t}, D\right) u=f
$$

Since the uniqueness is implied by Theorem 9.3, the assertion is proved.

As a consequence of Theorem 9.3, 9.4, and Theorem 10.2 we immediately have

10.3. Corollary. Let $s$ and $\varrho$ be as in Theorem 10.2. Then $A\left(\partial_{t}, D\right)$ is an isomorphism of $H_{(0), B}^{(s)}(Q ; \varrho)$ onto $H_{(0)}^{(s-\mu)}(Q ; \varrho)$, whose inverse will be denoted by $A\left(\partial_{t}, D\right)^{-1}$. 


\section{Existence and uniqueness theorem in the general case}

11.1. Let $s \in R$, and let $\varrho>0$ be as in Theorem 10.2 (or 10.1).

This section deals with the existence and uniqueness of solutions in $H^{(s)}(Q ; \varrho)$ to Problem (QP) in the general case, that is, for the data

$$
\begin{array}{ll}
f \in H^{(s-\mu)}(Q ; \varrho), & \\
\varphi_{k} \in H^{s-k q_{0}-q_{0} / 2}\left(\boldsymbol{R}_{+}^{n}\right), & k=0, \ldots, m_{0}-1, \\
g_{j} \in H^{\left(s-\mu_{j}-q_{n} / 2\right)}(\Sigma ; \varrho), \quad j=1, \ldots, \varkappa .
\end{array}
$$

Let us first define

by setting

$$
\Phi_{k} \in H^{s-k q_{0}-q_{0} / 2}\left(\boldsymbol{R}_{+}^{n}\right), \quad 0 \leqq k q_{0}<s-q_{0} / 2,
$$

$$
\Phi_{k}=\varphi_{k} \quad \text { for } \quad k=0, \ldots, m_{0}-1
$$

and (see 7.1)

$$
\Phi_{m_{0}+r}=T_{r} f+\sum_{v=0}^{m_{0}-1} S_{r-v} \sum_{k=0}^{m_{0}-1-v} A^{(k)}(D) \varphi_{v+k}
$$

when $0 \leqq r q_{0}<s-\mu-q_{0} / 2$.

Assume now that Problem (QP) has a solution in $H^{(s)}(Q ; \varrho)$. Then Theorem 7.2 implies that necessarily

$$
\left(\left(\Phi_{k}\right)_{k},\left(g_{j}\right)_{j}\right) \in E^{s}(\varrho) .
$$

For the converse we have the following result:

11.2. Theorem. Suppose that $s \in R_{q_{0}}$, and that $\varrho>0$ is given as in Theorem 10.2. Then Problem (QP) with the data 11.1(1)-11.1(3) has a solution in $H^{(s)}(Q ; Q)$ if (and only if)

$$
\left(\left(\Phi_{k}\right)_{k},\left(g_{j}\right)_{j}\right) \in E^{s}(\varrho),
$$

where the $\Phi_{k}$ are given by 11.1(4) and 11.1(5)

Moreover, there is at most one solution.

Proof. The sufficiency of condition (1).

11.2.1. Assume that condition (1) holds. Then there exists $v \in H^{(s)}(Q ; \varrho)$ such that

Now, setting

$$
\Psi v=\left(\left(\Phi_{k}\right)_{k},\left(g_{j}\right)_{j}\right)
$$

we have

$$
f_{v}=A\left(\partial_{t}, D\right) v \in H^{(s-\mu)}(Q ; \varrho)
$$

so that, by $11.1(5)$,

$$
\gamma_{t} \partial_{t}^{m_{0}+r} v=T_{r} f_{v}+\sum_{v=0}^{m_{0}-1} S_{r-v} \sum_{k=0}^{m_{0}-1-v} A^{(k)}(D) \varphi_{v+k},
$$

$$
T_{r} f=T_{r} f_{v}, \quad r q_{0}<s-\mu-q_{0} / 2 .
$$


Thus (see 7.1)

and hence

$$
\gamma_{t} \partial_{t}^{r} f-\sum_{k=0}^{m_{0}-1} A^{(k)}(D) T_{r-m_{0}+k} f=\gamma_{t} \partial_{t}^{r} f_{v}-\sum_{k=0}^{m_{0}-1} A^{(k)}(D) T_{r-m_{0}+k} f_{v}
$$

This in turn implies

$$
\gamma_{t} \partial_{t}^{r}\left(f-f_{v}\right)=0, \quad r q_{0}<s-\mu-q_{0} / 2
$$

$$
f-f_{v} \in H_{(0)}^{(s-\mu)}(Q ; \varrho) .
$$

11.2.2. We are now in a position to apply Theorem 10.2 and see that there is $w \in H_{(0), B}^{(s)}(Q ; \varrho)$ such that

$$
A\left(\partial_{t}, D\right) w=f-f_{v} .
$$

Consequently, the function $u=v+w \in H^{(s)}(Q ; \varrho)$ is a solution of Problem (QP).

It remains to prove the uniqueness of the solution.

11.2.3. Let us suppose that Problem (QP) has two solutions $u$ and $v$ in $H^{(s)}(Q ; \varrho)$. Then

satisfies the conditions

$$
w=u-v \in H^{(s)}(Q ; \varrho)
$$

$$
\begin{aligned}
& f_{w}=A\left(\partial_{t}, D\right) w=0, \\
& \psi_{k}=\gamma_{t} \partial_{t}^{k} w=0, \quad k=0, \ldots, m_{0}-1 .
\end{aligned}
$$

But by virtue of Theorem 7.2, we now have also

$$
\gamma_{t} \partial_{t}^{m_{0}+r_{W}}=T_{r} f_{w}+\sum_{k=0}^{m_{0}-1} S_{r-v} \sum_{k=0}^{m_{0}-1-v} A^{(k)}(D) \psi_{v+k}=0
$$

if $r q_{0}<s-\mu-q_{0} / 2$. Since furthermore

we conclude that

$$
\gamma_{n} B_{j}\left(\partial_{t}, D\right) w=0, \quad j=1, \ldots, \varkappa,
$$

$$
w \in H_{(0), B}^{(s)}(Q ; \varrho) .
$$

Thus Theorem 10.2 (or Theorem 9.3) implies $w=0$ and hence $u=v$.

\section{A priori estimates in $H^{(s)}(Q ; \varrho)$}

12.1. Let $s \in R$ and $\varrho>0$ be given. From 8.4 and 8.5 it immediately follows, by Theorem 8.3 , that

$$
\|f\|_{H^{(s-\mu)}(Q ; \varrho)}+\left\|\left(\left(\Phi_{k}\right)_{k},\left(g_{j}\right)_{j}\right)\right\|_{E^{s}(\varrho)} \leqq C\|u\|_{H^{(s)}(Q ; \varrho)}
$$

for all $u \in H^{(s)}(Q ; \varrho)$, where

$$
\begin{array}{ll}
f=A\left(\partial_{t}, D\right) u, & \\
\Phi_{k}=\gamma_{t} \partial_{t}^{k} u, & 0 \leqq k q_{0}<s-q_{0} / 2, \\
g_{j}=\gamma_{n} B_{j}\left(\partial_{t}, D\right) u, & j=1, \ldots, x .
\end{array}
$$


Setting

$$
\varphi_{k}=\Phi_{k} \quad \text { for } \quad k=0, \ldots, m_{0}-1,
$$

we have, by Theorem 7.2,

$$
\Phi_{m_{0}+r}=T_{r} f+\sum_{v=0}^{m_{0}-1} S_{r-v} \sum_{k=0}^{m_{0}-1-v} A^{(k)}(D) \varphi_{v+k}
$$

for $0 \leqq r q_{0}<s-\mu-q_{0} / 2$. This means that the left side of the estimate (1) actually depends only on $f,\left(\varphi_{k}\right)_{k}$, and $\left(g_{j}\right)_{j}$.

Next we shall study the validity of the reversed estimate of (1).

We begin by stating and proving the basic result of this section.

12.2. Theorem. Suppose $s \in R_{q_{0}}$, and let $\varrho>0$ be as in Theorem 10.2. Then the a priori estimate

$$
\|u\|_{H^{(s)}(Q ; \varrho)} \leqq C\left(\|f\|_{H^{(s-\mu)}(Q ; \varrho)}+\left\|\left(\left(\Phi_{k}\right)_{k},\left(g_{j}\right)_{j}\right)\right\|_{E^{s}(\ell)}\right)
$$

with the notation

$$
\begin{array}{ll}
f=A\left(\partial_{t}, D\right) u, & k=0, \ldots, m_{0}-1, \\
\Phi_{k}=\varphi_{k}=\gamma_{t} \partial_{t}^{k} u, & \\
\Phi_{m_{0}+r}=T_{r} f+\sum_{v=0}^{m_{0}-1} S_{r-v} \sum_{k=0}^{m_{0}-1-v} A^{(k)}(D) \varphi_{v+k}, & 0 \leqq r q_{0}<s-\mu-q_{0} / 2, \\
g_{j}=\gamma_{n} B_{j}\left(\partial_{t}, D\right) u, & j=1, \ldots, x,
\end{array}
$$

holds for all $u \in H^{(s)}(Q ; \varrho)$ if and only if the condition (RI) is satisfied:

(RI) The mapping

$$
\Psi: H^{(s)}(Q ; \varrho) \rightarrow E^{s}(\varrho)
$$

has a continuous linear right inverse $\Psi_{R}^{-1}$,

$$
\Psi \Psi_{R}^{-1} V=V, \quad V \in E^{s}(\varrho) .
$$

Proof. The sufficiency of condition (RI).

12.2.1. Let $u \in H^{(s)}(Q ; \varrho)$, and set

$$
V=\left(\left(\Phi_{k}\right)_{k},\left(g_{j}\right)_{j}\right) .
$$

Then $V \in E^{s}(\varrho)$, and we have, by assumption,

and

$$
v=\Psi_{R}^{-1} V \in H^{(s)}(Q ; \varrho)
$$

$$
\|v\|_{H^{(s)}(Q ; \varrho)} \leqq C\|V\|_{E^{s}(\varrho)} .
$$

If we now put

then

$$
w=u-v \in H^{(s)}(Q ; \varrho),
$$

$$
\gamma_{t} \partial_{t}^{k} w=0 \text { for } k q_{0}<s-q_{0} / 2
$$


and

so that

$$
\gamma_{n} B_{j}\left(\partial_{t}, D\right) w=0 \text { for } j=1, \ldots, \varkappa,
$$

$$
w \in H_{(0), B}^{(s)}(Q ; \varrho) .
$$

By virtue of Theorem 9.3 we thus obtain, using (1),

that is, the estimate (A).

$$
\begin{aligned}
\|u\|_{H^{(s)}(Q ; \varrho)} \leqq & C\|A w\|_{H^{(s-\mu)}(Q ; \varrho)}+\|v\|_{H^{(s)}(Q ; \varrho)} \\
& \leqq C\left(\|f\|_{H^{(s-\mu)}(Q ; \varrho)}+\|V\|_{E^{s}(\varrho)}\right),
\end{aligned}
$$

The necessity of condition (RI).

12.2.2. Let us suppose that

Then we have (see 8.5)

$$
V=\left(\left(\Phi_{k}\right)_{k},\left(g_{j}\right)_{j}\right) \in E^{s}(\varrho) .
$$

If we set

$$
v=\Psi_{0}^{-1} V \in H_{\Psi}^{(s)}(Q ; \varrho)^{\perp} .
$$

$$
f_{v}=A\left(\partial_{t}, D\right) v \in H^{(s-\mu)}(Q ; \varrho)
$$

and $\varphi_{k}=\Phi_{k}$ for $k=0, \ldots, m_{0}-1$, then

$$
\Phi_{m_{0}+r}=T_{r} f_{v}+\sum_{v=0}^{m_{0}-1} S_{r-v} \sum_{k=0}^{m_{0}-1-v} A^{(k)}(D) \varphi_{v+k}
$$

when $0 \leqq r q_{0}<s-\mu-q_{0} / 2$.

By Proposition 4.4, we now have

$$
\gamma_{t} \partial_{t}^{r} f_{v} \in H^{s-\mu-r q_{0}-q_{0} / 2}\left(\boldsymbol{R}_{+}^{n}\right), \quad r q_{0}<s-\mu-q_{0} / 2 .
$$

Thus Proposition 4.7 implies that

$$
f=\left(\gamma_{t}^{s-\mu}\right)_{R}^{-1} \gamma_{t}^{s-\mu} f_{v} \in H^{(s-\mu)}(Q ; \varrho)
$$

satisfies

$$
\|f\|_{H^{(s-\mu)}(Q ; \varrho)} \leqq C \sum_{r}\left\|\gamma_{t} \partial_{t}^{r} f_{v}\right\|_{H^{s-\mu-r q_{0}-q_{0} / 2\left(R_{+}^{n}\right)}} .
$$

It follows from (3) that $f-f_{v} \in H_{(0)}^{(s-\mu)}(Q ; \varrho)$. Therefore we have, by applying Corollary 10.3 to $f-f_{v}$,

$$
w=A\left(\partial_{t}, D\right)^{-1}\left(f-f_{v}\right) \in H_{(0), B}^{(s)}(Q ; \varrho) .
$$

12.2.3. Let us now set

$$
u=v+w \in H^{(s)}(Q ; \varrho) .
$$

Then

and

$$
A\left(\partial_{t}, D\right) u=f
$$

$$
\Psi u=V=\left(\left(\Phi_{k}\right)_{k},\left(g_{j}\right)_{j}\right)
$$


By (4), the estimate (A) therefore yields

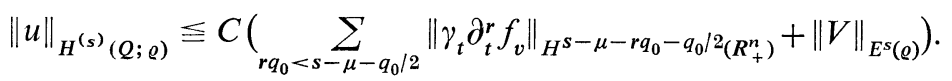

In view of 7.1 we have here

But by (2),

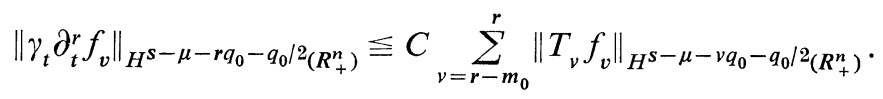

where (see 7.1)

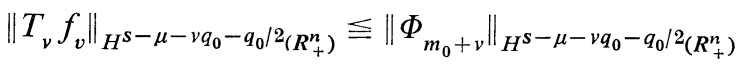

$$
\begin{aligned}
& +\sum_{\lambda=0}^{m_{0}-1} \sum_{k=0}^{m_{0}-1-\lambda}\left\|S_{\nu-\lambda} A^{(k)}(D) \varphi_{\lambda+k}\right\|_{H^{s-\mu-v q_{0}-q_{0} / 2}\left(R_{+}^{n}\right)} .
\end{aligned}
$$

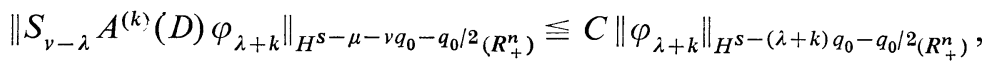

Thus we obtain

$$
\|u\|_{H^{(s)}(Q ; \varrho)} \leqq C\|V\|_{E^{s}(\varrho)} .
$$

Finally, combining (5) and (6), we see that the mapping

$$
V \mapsto u=v+w
$$

gives the desired inverse $\Psi_{R}^{-1}$, that is,

$$
\Psi_{R}^{-1}=\Psi_{0}^{-1}+A\left(\partial_{t}, D\right)^{-1}\left(\left(\gamma_{t}^{s-\mu}\right)_{R}^{-1} \gamma_{t}^{s-\mu} A\left(\partial_{t}, D\right)-A\left(\partial_{t}, D\right)\right) \Psi_{0}^{-1}
$$

This completes the proof.

12.3. Corollary. Under the same hypotheses and with the same notation as in Theorem 12.2, condition (RI) is satisfied if and only if the estimate

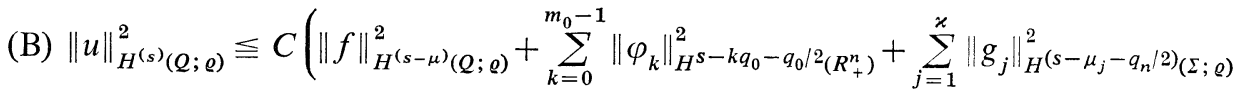

$\left.+\left.\sum_{\mu_{j}+v q_{0}=s-\delta} \int_{0}^{\infty} \int\right|_{k q_{0} \leqq \mu_{j}}\left(B_{j}^{(k)}(D) \Phi_{v+k}\right)\left(x^{\prime}, \sigma^{s / q_{0}}\right)-\left.\exp \left(-\varrho \sigma^{s / q_{n}}\right)\left(\partial_{t}^{v} g_{j}\right)\left(\sigma^{s / q_{n}}, x^{\prime}\right)\right|^{2} d x^{\prime} \frac{d \sigma}{\sigma}\right)$

holds for all $u \in H^{(s)}(Q ; \varrho)$.

Proof. By virtue of Theorem 12.2, it is enough to show that the estimate (A) implies (B) because (B) immediately implies (A). This, however, follows from the inequality (see 7.1)

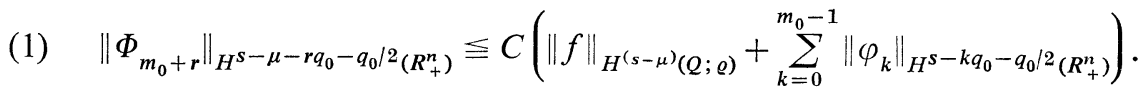

It is worthwhile to note the following special case: 
12.4. Corollary. Suppose that the hypotheses of Theorem 12.2 are satisfied, and that $s-\mu_{j} \neq \delta \bmod q_{0}$ for $j=1, \ldots, \varkappa$. Then

$$
\begin{aligned}
\|u\|_{H^{(s)}(Q ; \varrho)} \leqq & C\left(\left\|A\left(\partial_{t}, D\right) u\right\|_{H^{(s-\mu)}(Q ; \varrho)}\right. \\
& \left.+\sum_{k<m_{0}}\left\|\gamma_{t} \partial_{t}^{k} u\right\|_{H^{s-k q_{0}-q_{0} / 2}\left(R_{+}^{n}\right)}+\sum_{j}\left\|\gamma_{n} B_{j}\left(\partial_{t}, D\right) u\right\|_{H^{\left(s-\mu_{j}-q_{n} / 2\right)(\Sigma ; \varrho)}}\right)
\end{aligned}
$$

for all $u \in H^{(s)}(Q ; \varrho)$.

Proof. By Corollary 12.3, it suffices to prove that condition (RI) holds.

To do this, let

Then (see 8.5)

$$
V=\left(\left(\Phi_{k}\right)_{k},\left(g_{j}\right)_{j}\right) \in E^{s}(\varrho)
$$

and further

$$
v=\Psi_{0}^{-1} V \in H_{\Psi}^{(s)}(Q ; \varrho) \perp,
$$

$$
f=A\left(\partial_{t}, D\right) v \in H^{(s-\mu)}(Q ; \varrho) .
$$

By Propositions 4.4 and 4.7 we see that

and that

$$
f_{0}=\left(\gamma_{t}^{s-\mu}\right)_{R}^{-1} \gamma_{t}^{s-\mu} f \in H^{(s-\mu)}(Q ; \varrho),
$$

$$
\left\|f_{0}\right\|_{H^{(s-\mu)}(Q ; \varrho)} \leqq C \sum_{r}\left\|\gamma_{t} \partial_{t}^{r} f\right\|_{H^{s-\mu-r q_{0}-q_{0} / 2}\left(R_{+}^{n}\right)} .
$$

Now it follows from 7.1, Theorem 7.2, and Theorem 11.2 that the problem

$$
\begin{array}{ll}
A\left(\partial_{t}, D\right) u=f_{0}, & \\
\gamma_{t} \partial_{t}^{k} u=\varphi_{k}=\Phi_{k}, & k=0, \ldots, m_{0}-1, \\
\gamma_{n} B_{j}\left(\partial_{t}, D\right) u=g_{j}, & j=1, \ldots, \varkappa,
\end{array}
$$

has a unique solution $u \in H^{(s)}(Q ; \varrho)$ which satisfies also

$$
\Psi u=V .
$$

Next, again by Propositions 4.4 and 4.7, we know that

with

$$
v_{0}=\left(\gamma_{t}^{s}\right)_{R}^{-1}\left(\Phi_{k}\right)_{k} \in H^{(s)}(Q ; \varrho)
$$

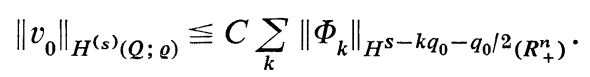

If we now set $w=u-v_{0}$, then

and Theorem 9.2 gives

$$
w \in H_{(0)}^{(s)}(Q ; \varrho),
$$

$$
\|w\|_{H^{(s)}(Q ; \varrho)} \leqq C\left(\left\|A\left(\partial_{t}, D\right) w\right\|_{H^{(s-\mu)}(Q ; \varrho)}+\sum_{j}\left\|\gamma_{n} B_{j}\left(\partial_{t}, D\right) w\right\|_{H^{\left(s-\mu_{j}-q_{n} / 2\right)(\Sigma ; \varrho)}}\right) .
$$

Therefore

$$
\|u\|_{H^{(s)}(Q ; \varrho)} \leqq C\left(\left\|v_{0}\right\|_{H^{(s)}(Q ; \varrho)}+\left\|f_{0}\right\|_{H^{(s-\mu)}(Q ; \varrho)}+\sum_{i}\left\|g_{j}\right\|_{H^{\left(s-\mu_{j}-q_{n} / 2\right)(\Sigma ; \varrho)}}\right) .
$$


Hence it follows from (1) and (2) that

where, by 7.1 ,

$$
\begin{aligned}
& \|u\|_{H^{(s)}(\underline{Q} ; \varrho)} \leqq C\left(\sum_{k}\left\|\Phi_{k}\right\|_{H^{s-k q_{0}-q_{0} / 2\left(R_{+}^{n}\right)}}+\sum_{j}\left\|g_{j}\right\|_{H^{\left(s-\mu_{j}-q_{n} / 2\right)(\Sigma ; \varrho)}}\right. \\
& +\sum_{r}\left\|\gamma_{t} \partial_{t}^{r} f\right\|_{\left.H^{s-\mu-r q_{0}-q_{0} / 2\left(R_{+}^{n}\right)}\right)}
\end{aligned}
$$

Thus we have

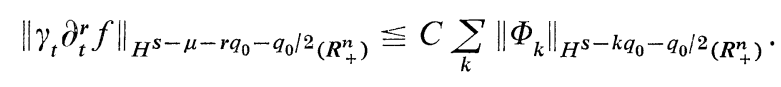

$$
\|u\|_{H^{(s)}(Q ; \varrho)} \leqq C\|V\|_{E^{s}(\varrho)},
$$

and a reasoning quite analogous to the one used in 12.2.3 completes the proof.

Remark. One can also prove the assertion, more directly and a little more briefly, by applying Theorem 9.2 to $w=u-v_{0}$ ( $u$ is arbitrary and $v_{0}$ as above) to obtain (3), and by making then use of (2) and the inequality $12.3(1)$.

\section{The case of normal boundary operator system $\left\{B_{j}(D)\right\}$}

This section is devoted to an important special case in order to illustrate the substance of the results of the preceding sections (see Conclusion 13.6).

The boundary operators $B_{j}, j=1, \ldots, x$, with which we shall deal here are of the form

$$
B_{j}=B_{j}(D)=\sum_{\langle\alpha, q\rangle \leqq \mu_{j}} b_{j \alpha} D^{\alpha} .
$$

We notice that $q_{n} \mid \mu_{j}$ (i.e., $\mu_{j}$ is a multiple of $q_{n}$ ) if and only if $B_{j}(D)$ is formally normal (to $R^{n-1}$ ) of order $\mu_{j}$ :

$$
B_{j}(D)=\beta_{j} D_{n}^{v_{j}}+B_{j}^{00}(D),
$$

where $v_{j}=\mu_{j} / q_{n}, \beta_{j}=b_{j,\left(0, v_{j}\right)}$, and the order of $B_{j}^{00}(D)$ with respect to $D_{n}$ is less than $\mu_{j}$.

The system $\left\{B_{j}(D)\right\}$ of the operators $B_{1}(D), \ldots, B_{\varkappa}(D)$ is called normal (to $R^{n-1}$ ) if

(i) $q_{n} \mid \mu_{j}$ and $\beta_{j} \neq 0 \quad$ for $j=1, \ldots, x$;

(ii) $\mu_{j} \neq \mu_{k}$ (or $v_{j} \neq v_{k}$ ) for $j \neq k$.

In what follows we assume (explicitly but superfluously, in fact) that the system $\left\{B_{1}(D), \ldots, B_{\varkappa}(D)\right\}$ is normal, and that $s>\max \left\{\mu_{j}\right\}+q_{n} / 2, \varrho>0$.

13.1. Lemma. The mapping

$$
\left(\gamma_{n} B_{j}(D)\right)_{j}: H^{(s)}(Q ; \varrho) \rightarrow \prod_{j} H^{\left(s-\mu_{j}-q_{n} / 2\right)}(\Sigma ; \varrho)
$$

is a continuous surjective operator which has a continuous linear right inverse $B_{R}^{-1}$. 
Proof. Assume first that $F_{k}(D), 0 \leqq k q_{n}<s-q_{n} / 2$, are operators of orders $k q_{n}$, respectively. By Proposition 4.6 one can then show that the operator

$$
\left(\gamma_{n} F_{k}(D)\right)_{k q_{n}<s-q_{n} / 2}: H^{(s)}(Q ; \varrho) \rightarrow \prod_{k} H^{\left(s-k q_{n}-q_{n} / 2\right)}(\Sigma ; \varrho)
$$

has a continuous linear right inverse $F_{R}^{-1}$.

Let us now choose

$$
\begin{array}{ll}
F_{k}(D)=B_{j}(D) & \text { if } \quad k=v_{j}=\mu_{j} / q_{n}, \\
F_{k}(D)=D_{n}^{k} & \text { otherwise. }
\end{array}
$$

Then we can define $B_{R}^{-1}$ as the restriction of $F_{R}^{-1}$ to $\prod_{j} H^{\left(s-\mu_{j}-q_{n} / 2\right)}(\Sigma ; \varrho)$.

13.2. From Theorems 8.2 and 8.3 we see that every element of $E^{s}(\varrho)$ satisfies the compatibility relations (LCR) and (GCR) of 8.4. These conditions now characterize the space $E^{s}(\varrho)$ completely. Indeed, we have

13.3. Theorem. $E^{s}(\varrho)=F^{s}(\varrho)$.

Proof. In view of the definition of $E^{s}(\varrho)$ we have to show that $F^{s}(\varrho) \subset E^{s}(\varrho)$ (see 8.4 and 8.5 ).

13.3.1. Assume that

Using Lemma 13.1 we set

$$
\left(\left(\Phi_{k}\right)_{k},\left(g_{j}\right)_{j}\right) \in F^{s}(\varrho)
$$

$$
v=B_{R}^{-1}\left(g_{j}\right)_{j} \in H^{(s)}(Q ; \varrho)
$$

Define furthermore by

$$
\psi_{k}, \chi_{k} \in H^{s-k q_{0}-q_{0} / 2}\left(\boldsymbol{R}_{+}^{n}\right), \quad 0 \leqq k q_{0}<s-q_{0} / 2,
$$

$$
\psi_{k}=\gamma_{t} \partial_{t}^{k} v, \quad \chi_{k}=\Phi_{k}-\psi_{k} .
$$

13.3.2. Let $H_{B}^{s}\left(\boldsymbol{R}_{+}^{n}\right)$ denote the closed subspace of $H^{s}\left(\boldsymbol{R}_{+}^{n}\right)$ which is given by

$$
H_{B}^{s}\left(\boldsymbol{R}_{+}^{n}\right)=\left\{\chi \in H^{s}\left(\boldsymbol{R}_{+}^{n}\right) \mid \gamma_{n} B_{j}(D) \chi=0, j=1, \ldots, \chi\right\} .
$$

Then by (an analogue of) a result of P. Grisvard (see [2], Théorème 8.1) we have:

13.3.3. Proposition. For any $\theta, 0<\theta<1,\left[H_{B}^{s}\left(\boldsymbol{R}_{+}^{n}\right), H^{0}\left(\boldsymbol{R}_{+}^{n}\right)\right]_{\theta}$ is a vector subspace of $H^{s(1-\theta)}\left(\boldsymbol{R}_{+}^{n}\right)$ consisting of functions $\chi$ such that

(i) $\gamma_{n} B_{j}(D) \chi=0 \quad$ if $\mu_{j}<s(1-\theta)-q_{n} / 2$;

(ii) $x_{n}^{-1 / 2} B_{j}(D) \chi \in L^{2}\left(\boldsymbol{R}_{+}^{n}\right)$ if $\mu_{j}=s(1-\theta)-q_{n} / 2$.

The square of the norm of $\chi \in\left[H_{B}^{s}\left(\boldsymbol{R}_{+}^{n}\right), H^{0}\left(\boldsymbol{R}_{+}^{n}\right)\right]_{\theta}$ can be defined by

$$
\|\chi\|_{H^{s(1-\theta)}\left(R_{+}^{n}\right)}^{2}+\sum_{\mu_{j}=s(1-\theta)-q_{n} / 2} \int_{0}^{\infty} \int\left|B_{j}(D) \chi\left(x^{\prime}, \sigma\right)\right|^{2} d x^{\prime} \frac{d \sigma}{\sigma} .
$$


13.3.4. We now have

so that

$$
\left(\left(\psi_{k}\right)_{k},\left(g_{j}\right)_{j}\right) \in E^{s}(\varrho),
$$

Hence (see 8.4)

$$
\left(\left(\chi_{k}\right)_{k},(0)_{j}\right) \in F^{s}(\varrho)
$$

and

$$
\gamma_{n} B_{j}(D) \chi_{k}=0 \quad \text { if } \quad \mu_{j}+k q_{0}<s-\delta
$$

$$
\int_{0}^{\infty} \int\left|B_{j}(D) \chi_{k}\left(x^{\prime}, \sigma\right)\right|^{2} d x^{\prime} \frac{d \sigma}{\sigma}<\infty \quad \text { if } \quad \mu_{j}+k q_{0}=s-\delta .
$$

Thus Proposition 13.3.3 implies that

$$
\chi_{k} \in\left[H_{B}^{s}\left(\boldsymbol{R}_{+}^{n}\right), H^{0}\left(\boldsymbol{R}_{+}^{n}\right)\right]_{\theta_{k}} \text { with } \theta_{k}=\left(k q_{0}+q_{0} / 2\right) / s .
$$

13.3.5. Let $\{X, Y\}$ be an interpolation couple of Hilbert spaces $X$ and $Y$. One can then show, for example by using methods similar to those in [4], Chap. I, $\S 3,4$, that the trace mapping

$$
\left(\gamma_{t} \partial_{t}^{k}\right)_{k q_{0}<s-q_{0} / 2}: H^{s}\left(\boldsymbol{R}_{+} ; \varrho ; Y\right) \cap H^{0}\left(\boldsymbol{R}_{+} ; \varrho ; X\right) \rightarrow \prod_{k}[X, Y]_{\left(k q_{0}+q_{0} / 2\right) / s}
$$

is a continuous surjective operator which has a continuous linear right inverse.

Take now

$$
X=H_{B}^{s}\left(\boldsymbol{R}_{+}^{n}\right), \quad Y=H^{0}\left(\boldsymbol{R}_{+}^{n}\right),
$$

denote the above-mentioned inverse by $I_{R}$, and define

$$
w=I_{R}\left(\chi_{k}\right)_{k} .
$$

Then $w \in H^{(s)}(Q ; \varrho)$ and

13.3.6. Let us now set

$$
\Psi_{w}=\left(\left(\chi_{k}\right)_{k},(0)_{j}\right)
$$

Then it is evident that

$$
u=v+w \in H^{(s)}(Q ; \varrho) .
$$

Consequently,

$$
\Psi u=\left(\left(\Phi_{k}\right)_{k},\left(g_{j}\right)_{j}\right) .
$$

and the proof is complete.

$$
\left(\left(\Phi_{k}\right)_{k},\left(g_{j}\right)_{j}\right) \in E^{s}(\varrho),
$$

13.4. Keeping Theorem 12.2 in mind, we are next going to show that condition (RI) is satisfied in the case considered.

13.5. The orem. The operator

$$
\Psi: H^{(s)}(Q ; \varrho) \rightarrow E^{s}(\varrho)
$$

has a continuous linear right inverse $\Psi_{R}^{-1}$. 
Proof. By making use of Lemma 13.1, the proof of Theorem 13.3, and its notation, we set, for every $V=\left(\left(\Phi_{k}\right)_{k},\left(g_{j}\right)_{j}\right) \in E^{s}(\varrho)$,

$$
\begin{aligned}
\Psi_{R}^{-1} V & =I_{R}\left(\Phi_{k}-\gamma_{t} \partial_{t}^{k} B_{R}^{-1}\left(g_{j}\right)_{j}\right)_{k}+B_{R}^{-1}\left(g_{j}\right)_{j} \\
& =I_{R}\left(\chi_{k}\right)_{k}+B_{R}^{-1}\left(g_{j}\right)_{j} .
\end{aligned}
$$

We claim that this definition yields what we want.

First of all, $\Psi_{R}^{-1}$ is a linear mapping of $E^{s}(\varrho)$ into $H^{(s)}(Q ; \varrho)$ satisfying

$$
\Psi \Psi_{R}^{-1} V=V, \quad V \in E^{s}(\varrho) .
$$

To prove the continuity of $\Psi_{R}^{-1}$, let us consider the inequality

$$
\left\|\Psi_{R}^{-1} V\right\|_{H^{(s)}(Q ; \varrho)} \leqq\left\|I_{R}\left(\chi_{k}\right)_{k}\right\|_{H^{(s)}(Q ; \varrho)}+\left\|B_{R}^{-1}\left(g_{j}\right)_{j}\right\|_{H^{(s)}(Q ; \varrho)} .
$$

From 13.3.5 and Proposition 13.3.3 it follows that

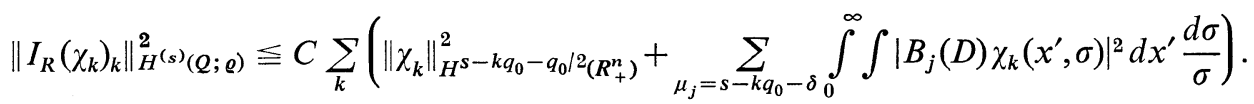

Here we have, by 13.3.1 and Theorem 13.1,

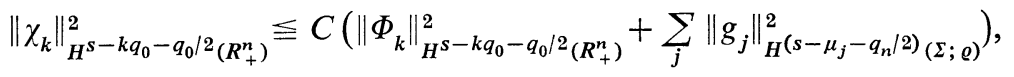

and by Theorem 8.3, 13.3.1, and Lemma 13.1,

$$
\begin{aligned}
& \int_{0}^{\infty} \int\left|B_{j}(D) \chi_{k}\left(x^{\prime}, \sigma\right)\right|^{2} d x^{\prime} \frac{d \sigma}{\sigma} \\
& \leqq C\left(\int_{0}^{\infty} \int\left|\left(B_{j}(D) \Phi_{k}\right)\left(x^{\prime}, \sigma^{s / q_{0}}\right)-\exp \left(-\varrho \sigma^{s / q_{n}}\right)\left(\partial_{t}^{k} g_{j}\right)\left(\sigma^{s / q_{n}}, x^{\prime}\right)\right|^{2} d x^{\prime} \frac{d \sigma}{\sigma}\right. \\
&\left.+\sum_{j}\left\|g_{j}\right\|_{\left.H^{\left(s-\mu_{j}-q_{n} / 2\right)_{(\Sigma ; \varrho)}}\right)}^{2}\right) .
\end{aligned}
$$

Therefore

$$
\left\|I_{R}\left(\chi_{k}\right)_{k}\right\|_{H^{(s)}(Q ; \varrho)} \leqq C\left\|\left(\left(\Phi_{k}\right)_{k},\left(g_{j}\right)_{j}\right)\right\|_{E^{s}(\varrho)} .
$$

Thus we see, in view of Lemma 13.1, that

$$
\left\|\Psi_{R}^{-1} V\right\|_{H^{(s)}(Q ; \varrho)} \leqq C\|V\|_{E^{s}(\varrho)} .
$$

Remark. The existence of a continuous linear right inverse of $\Psi$ follows also from the fact that, by virtue of $8.4,8.5$, and Theorem $13.3, \Psi$ is now a continuous surjective operator of $H^{(s)}(Q ; \varrho)$ onto the Hilbert space $E^{s}(\varrho)=F^{s}(\varrho)$.

\subsection{Conclusion.}

13.6.1. Combining Theorems 11.2 and 13.3 we see that, under the hypotheses of Theorem 11.2, Problem (QP) has a (unique) solution in $H^{(s)}(Q ; \varrho)$ if and only if conditions (LCR) and (GCR) of 8.4 are satisfied.

13.6.2. Combining Theorem 13.5 with Theorem 12.2 and Corollary 12.3, we reach the conclusion that, under the hypotheses of Theorem 12.2, the estimates 12.2(A) and 12.3(B), respectively, are always valid. 


\title{
References
}

[1] Agranovič, M. S., and M. I. VIšıK: Elliptic problems with a parameter and parabolic problems of general type. - Russian Math. Surveys 19, 1964, no. 3, 53-157.

[2] Grisvard, P.: Caractérisation de quelques espaces d'interpolation. - Arch. Rational Mech. Anal. 25, 1967, 40-63.

[3] Hewitt, E., and K. Stromberg: Real and abstract analysis - Springer-Verlag, Berlin-Heidelberg-New York, 1965.

[4] Lions, J. L., and E. MAGENes: Non-homogeneous boundary value problems and applications. Volume I. - Die Grundlehren der mathematischen Wissenschaften 181. SpringerVerlag, Berlin-Heidelberg-New York, 1972.

[5] Lions, J. L., and E. MAGenes: Non-homogeneous boundary value problems and applications. Volume II. - Die Grundlehren der mathematischen Wissenschaften 182. SpringerVerlag, Berlin-Heidelberg-New York, 1972.

[6] Purmonen, V. T.: On parametrically quasi-elliptic boundary problems. - Ann. Acad. Sci. Fenn. Ser. A I 5, 1980, 237-262.

[7] Schwartz, L.: Méthodes mathématiques pour les sciences physiques. - [2 ${ }^{e}$ Édition revue et corrigée.] Hermann, Paris, 1965.

[8] Slobodeckĭ, L. N.: Generalized Sobolev spaces and their application to boundary problems for partial differential equations. - Amer. Math. Soc. Transl. (2) 57, 1966, 207-275.

[9] Troisi, M.: Problemi al contorno con condizioni omogenee per le equazioni quasi-ellittiche. - Ann. Mat. Pura Appl. (4), 90, 1971, 331-412.

\author{
University of Jyväskylä \\ Department of Mathematics \\ Seminaarinkatu 15 \\ SF-40100 Jyväskylä 10 \\ Finland
}

Received 19 March 1980 